\title{
Brain perivascular space imaging across the human lifespan
}

\author{
Kirsten M. Lynch ${ }^{1 *}$, Farshid Sepehrband ${ }^{1}$, Arthur W. Toga ${ }^{1}$, Jeiran Choupan ${ }^{1}$ \\ ${ }^{1}$ Laboratory of Neuro Imaging (LONI), USC Mark and Mary Stevens Institute for Neuroimaging and \\ Informatics, USC Keck School of Medicine, Los Angeles, CA, 90033 \\ *Correspondence to kirsten.lynch@loni.usc.edu
}

\begin{abstract}
Enlarged perivascular spaces (PVS) are considered a biomarker for vascular pathology and has been observed in several neurological disorders and in normal aging; however, the age-related changes in PVS structure across the normative lifespan are not well understood. We sought to characterize the relationship between PVS anatomical characteristics and age in a large cohort $(\sim 1400)$ between 8 and 90 years of age from the Human Connectome Project (HCP) Lifespan datasets. PVS from the white matter regions and the basal ganglia (BG) were automatically segmented and quantified from vesselness maps derived from multimodal structural MRI data. PVS burden increased from childhood to advancing age in both the white matter and BG and was reflected by increasing PVS volume fraction (VF), count and mean cross-sectional diameter and decreasing mean solidity, with elevated PVS burden in males compared to females. Within white matter regions, PVS burden in childhood significantly predicted the rate of PVS expansion across the lifespan $\left(t(66)=-12.15, p<.001, R^{2}=.69\right)$, where regions with low PVS VF in childhood undergo rapid growth (e.g., temporal regions) and regions with high PVS VF in childhood undergo slowed growth (e.g., limbic regions). PVS anatomical alterations across the lifespan was not significantly associated with working memory performance. Together, our findings of spatially-varying patterns of PVS enlargement across the lifespan constitutes a feature of normal aging and may reflect alterations to waste clearance functionality. These results provide a normative reference for the spatial distribution of PVS enlargement patterns from which pathological alterations can be compared.
\end{abstract}

\section{Significance Statement}

Perivascular spaces (PVS) surround penetrating blood vessels and play an important role in the brain waste clearance system. Enlarged PVS are observed in normal aging and neurological conditions; however, research on the role of PVS in health and disease are hindered by the lack of knowledge regarding the time course of PVS alterations across the lifespan. To address this, we characterized age-related alterations in PVS structure from childhood through advancing age. We found PVS become progressively wider and more numerous with differing regional trajectories. These findings provide an important step towards understanding the evolution of brain clearance mechanisms across the lifespan and can be used as a normative reference to better understand neurological disorders characterized by abnormal waste clearance.

Keywords: Perivascular spaces, waste clearance, lifespan, morphology, neuroimaging, cerebrovasculature 


\section{Introduction}

The brain waste clearance system consists of a network of vasculature that plays a critical role in the removal of toxic substrates from the brain to maintain tissue homeostasis. A major component of the waste clearance system includes perivascular spaces (PVS), which consist of tubular, interstitial fluid-filled cavities that surround small penetrating vessels in the brain parenchyma (1). PVS provide a low-resistance pathway to facilitate fluid exchange (2), where they accommodate the influx of cerebrospinal fluid (CSF) and energy substrates in the peri-arterial space and the drainage of interstitial fluid and metabolic waste through the peri-venous space (35). There is accumulating evidence that suggests pathological PVS enlargement is associated with reduced waste clearance functionality and can thus impede the removal of toxic metabolites, including amyloid beta $(A \beta)$, and render the brain susceptible to neurological damage (6). Because several neurological conditions are characterized by dysfunctional waste clearance ( 1 , $7,8)$, an understanding of the factors that contribute to PVS alterations can provide insight into disease pathogenesis.

Over the past decade, technological advancements in MRI acquisition and data processing have facilitated the study of PVS characteristics in health and neurological disease. Recent evidence from MRI studies in humans have shown increased PVS visibility in the brains of patients with neurological conditions, including multiple sclerosis (9) traumatic brain injury (10), stroke (11), Alzheimer's Disease (12), mild cognitive impairment (13) and Parkinson's disease (14). While typically considered a biomarker of vascular neuropathology, enlarged PVS are also observed in healthy, cognitively normal individuals. Increased PVS visibility is a prominent feature of advancing age $(15,16)$ and recent evidence has shown that enlarged PVS are also observed in typically developing adolescents (17) and young adults (18), albeit at much lower levels. However, the precise trajectory of PVS alterations as a consequence of age across the normative lifespan has yet to be fully described. Because the highest risk factor for the development of neurodegenerative disease is advancing age (19), age-related alterations to the components that facilitate the elimination of waste may render the brain more vulnerable to neurodegenerative pathology. Therefore, characterization of the time course of PVS alterations in the normal human brain can provide insight into the evolution of waste clearance mechanisms and can offer a benchmark from which pathological PVS enlargement can be differentiated from that attributed to normal, healthy aging.

PVS have been observed in several regions of the brain, including the centrum semiovale of the white matter, basal ganglia (BG), hippocampus and midbrain structures (20). PVS enlargement in these structures, particularly within the white matter and BG, are differentially associated with neurological conditions. Vasculopathies, such as cerebral small vessel disease (CSVD) and hypertension, are preferentially associated with increased PVS visibility in the BG (11, 20-23), while white matter PVS alterations are predominantly observed in patients with amyloidopathies $(22,24-26)$ and neuroinflammatory conditions, such as multiple sclerosis $(27$, 28). The apparent spatial dependence of neuropathological PVS enlargement suggest the mechanisms that contribute to increased PVS visibility differ between the white matter and BG. The heterogeneous characteristics of MRI-visible PVS therefore suggest that the mechanisms that govern PVS dilations in health and disease may differ according to spatial location. A comprehensive understanding of regional patterns of PVS enlargement across the lifespan in the BG and subcortical white matter would provide granular insight into brain regions that may be particularly susceptible to age-related changes in waste clearance processes. 
Much of the evidence of age-related changes to PVS burden come from studies that employ a visual rating scale to score the severity of PVS burden on select axial slices (15, 2933). While this approach can be easily implemented in clinical settings, it does not objectively quantify PVS content and may fail to capture regional PVS heterogeneity across the brain. Recently, efforts that utilize automated methods have enabled quantification of global PVS features; however, studies of normative aging have largely focused on PVS total volume or the fraction of tissue volume occupied by PVS (volume fraction, VF) $(18,34)$. These approaches provide limited information regarding the anatomical characteristics of PVS and can be confounded by total brain volume and age-related atrophy, as previous studies have shown PVS volume is significantly correlated with intracranial volume (ICV) $(18,34)$. Therefore, utilization of multiple morphological and structural characteristics, such as cross-sectional diameter, solidity and count, can provide greater insight into the mechanisms that contribute to age-related alterations in PVS volume.

The goal of this study is to characterize the influence of age on regional PVS burden in the white matter and BG across the lifespan in a large cross-sectional cohort ( 1400) of typically developing and cognitively normal children, adults and the elderly between 8 and 90 years of age. Here, we visualize PVS using an automated processing workflow to identify and quantify PVS morphometric features from vesselness maps derived from multi-modal structural neuroimaging contrasts designed to enhance PVS visibility (35). Features of PVS morphology, including PVS $\mathrm{VF}$, count, mean cross-sectional diameter and mean solidity, were extracted and used to quantify the magnitude and timing of PVS alterations across the lifespan in the BG and white matter regions using growth models. Additionally, we explored the influence of sex on age-related alterations to PVS morphology. Lastly, we relate variations in the PVS trajectory to working memory performance, a generalized behavioral domain indicative of fluid intelligence (36), in order to assess the functional implications of age-related PVS alterations on higher-order cognition. The results from this study will provide a normative reference for the spatial distribution and time course of PVS alterations across the lifespan, from which pathological alterations can be compared.

\section{Results}

Age distributions for the HCP cohorts stratified by sex are provided in Table 1. Overall, age was not significant different between sexes $(t(1394)=.79, p=.43)$; however females were significantly older than males in the HCP-YA cohort $(t(403)=-4.07, p<.001)$.

PVS alterations across the lifespan

PVS were present throughout the white matter and BG in all 3 cohorts and showed increasing presence with age (Fig. 1).

\section{Age-related PVS alterations in the basal ganglia}

Cohort-stratified multiple regression analyses show PVS morphological features are significantly associated with age after controlling for covariates in the HCP-A group only (Table 2). Age is positively associated with PVS VF $(p<.0001)$, PVS count $(p<.0001)$, and mean PVS diameter $(p<.0001)$ and negatively associated with mean PVS solidity $(p<.0001)$ in the HCP-A cohort (SI Appendix, Fig. S1). In the combined lifespan analyses, a quadratic regression best explained the age-related variance in PVS morphology (Table 3). The relationship between age 
and PVS VF, mean PVS diameter and PVS count were described with a convex curve (Fig. 2AC) and the relationship between age and mean PVS solidity were described with a concave curve (Fig. 2D). The minimum PVS volume fraction and PVS count are estimated at ages $14 \pm 3$ years and $43 \pm 2$ years, respectively and increase thereafter, while the maximum PVS mean solidity is estimated at age $10 \pm 9$ years and decreases nonlinearly. Across all features, the most rapid feature differences with age occur in the aging cohort (Fig. 2E).

Age-related PVS alterations in the total subcortical white matter

Cohort-stratified multiple regression analyses of PVS morphology in white matter show significant associations with age in multiple HCP cohorts (Table 2). PVS VF was significantly and positively associated with age in HCP-D $(p<.0001)$, HCP-YA $(p=.0002)$ and HCP-A $(p<.0001)$. Age was significantly associated with increasing PVS count in HCP-D $(p<.0001)$, but not HCP-A $(p=.40)$. The relationship between age and PVS count in the HCP-YA $(p=.0047)$ was reduced to non-significance following multiple comparison correction with a Bonferroni corrected threshold of $p<.0042$. Age was significantly associated with increased mean PVS diameter in HCP-A $(p<.0001)$, but not HCP-D $(p=.87)$ or HCP-YA following multiple comparison correction $(p=.0053)$. Mean PVS solidity was significantly and negatively associated with age in HCP-A $(p<.0001)$ and HCP-YA $(p=.0014)$, but not HCP-D $(p=.26)$. When combined across cohorts (Table 3$)$, a linear regression account for the most age-related variance in PVS VF (Fig. 3A) and mean PVS diameter (Fig. 3C). Age-related changes to PVS count were best described by a concave quadratic model with the most rapid changes observed early in life and peaking at $61 \pm 2$ years of age (Fig. 3B). Age-related reductions to PVS mean solidity were best described by a convex quadratic model that reaches stasis later in life with the estimated minimum solidity beyond the age range sampled $(90 \pm 25$ years) (Fig. 3D). Standardization of PVS features show all morphological characteristics contribute to the age-related changes to PVS VF with varying degrees (Fig. 3E).

Age-related PVS alterations within regions of the subcortical white matter

The distribution of PVS burden across regions per HCP cohort is shown in Fig. 4. Within HCP-D, temporal white matter has the lowest PVS VF $(M \pm S D=.0048 \pm .0027)$, particularly within parahippocampal and entorhinal regions, followed by occipital (.0051 \pm .0022$)$, parietal $(.0077 \pm .0035)$ and frontal $(.0071 \pm .0044)$ structures. The limbic white matter has the highest PVS VF in HCP-D (.0143 \pm .0047$)$, specifically within bilateral isthmus and rostral anterior cingulate regions. Within HCP-YA, occipital white matter has the lowest PVS VF (.0062 \pm .0028$)$, following by temporal (.0076 \pm .0034$)$, parietal $(.0100 \pm .0042)$, frontal $(.0119 \pm .0047)$ and limbic $(.0164 \pm .0046)$ structures. This trend was also observed in the HCP-A cohort (occipital: .0099 \pm .0039 ; temporal:

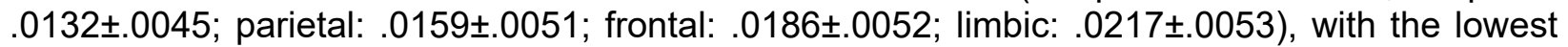
PVS VF in bilateral cuneus, parahippocampal and entorhinal regions and highest PVS VF in bilateral insula, rostral anterior cingulate and lateral orbitofrontal regions.

Exponential growth curves were used to quantify regional patterns of age-related PVS VF trajectories (Fig. 5). We found regions with the highest PVS VF in childhood are characterized by the slowest growth rate, including the white matter underlying the caudal anterior, isthmus and rostral anterior cingulate cortices bilaterally (Fig. 5A-B). Conversely, regions with low PVS VF in childhood, including white matter adjacent to bilateral cuneus, lateral and transverse occipital, inferior frontal and transverse temporal cortices had the largest growth rates. Indeed, we found the estimated PVS VF at age 8 years of age was significantly associated with slower growth rates 
across the lifespan (Fig. 5C; $B=-.71, t(66)=-12.15, p<.001, R^{2}=.69$ ), where limbic regions tend to have high PVS VF in childhood that changes minimally with age and temporal regions have low PVS VF in childhood that undergo the largest percent change across the lifespan (Fig. 6). Occipital regions deviate from this trend, where they have low PVS VF in childhood that remains low across the lifespan. These trends were observed bilaterally and the growth rate of left hemisphere white matter regions were significantly and positively correlated with the right hemisphere (Fig. 5D; $\left.B=.98, t(32)=16.43, p<.001, R^{2}=.89\right)$.

\section{PVS sex differences}

The main effect of sex on PVS morphological features are shown in Table 4. After controlling for age, scanner type and BG volume, a main effect of sex on PVS VF $(F(1,1388)=76.73, p<.0001)$, PVS count $(F(1,1388)=106.31, p<.0001)$, and mean PVS solidity $(F(1,1388)=77.85, p<.0001)$ were observed in the BG, where males had significantly larger PVS VF and count and smaller mean PVS solidity compared to females. The main effect of sex on mean PVS diameter in the BG did not survive multiple comparison correction $(F(1,1388)=7.27$, $p=.007)$. Within white matter, a significant effect of sex on mean PVS solidity $(F(1,1388)=63.83$, $p<.0001)$ and PVS VF $(F(1,1388)=24.51, p<.0001)$ was observed, where males had larger PVS VF and smaller mean PVS solidity compared to females after controlling for age, scanner type and total white matter volume. The main effect of sex on white matter PVS count $(F(1,1388)=5.36$, $p=.02)$, and mean diameter $(F(1,1388)=4.64, p=.03)$ did not survive multiple comparison correction.

While PVS burden in the BG was consistently larger in males compared to females across the lifespan (SI Appendix, Fig. S2), differences in the age-related trajectories of PVS morphological alterations were observed between sexes in the white matter (SI Appendix, Fig. S3). After controlling for covariates, a significant interaction between age and sex on white matter PVS VF $(F(3,1388)=17.62, p<.0001)$ was observed, such that the covariate-adjusted PVS VF rate of change was greater in females $\left(\beta=2.13 \times 10^{-4}, t(782)=34.30, \quad p<.0001\right.$, adjusted $\left.R^{2}=.60\right)$, compared to males $\left(\beta=1.75 \times 10^{-4}, t(606)=24.11, \quad p<.0001\right.$, adjusted $\left.R^{2}=.49\right)$. Similarly, an interaction between age and sex on PVS diameter was also observed $(F(3,1388)=21.95$, $p<.0001)$, where the adjusted mean diameter increased faster with age in females $(\beta=.014$, $t(782)=20.53, p<.0001$, adjusted $\left.R^{2}=.34\right)$, compared to males $(\beta=.009, t(606)=11.14, p<.0001$, adjusted $R^{2}=.17$ ). The cohort-stratified analyses also show males have significantly greater PVS VF and larger mean diameters compared to females in HCP-D and HCP-YA, while no significant sex differences were observed in HCP-A (Table 4). No significant interactions between age and sex were observed for the remaining PVS features.

\section{PVS associations with working memory}

In subjects between the ages of 12 and 59 years of age, we found the standardized score on the NIH Toolbox List Sorting Task decreases significantly with age after controlling for sex and scanner type (Fig. 7A; $t(1392)=-3.82, p=.0001)$. Age-adjusted standardized scores were used in subsequent analyses that assess the association between working memory and age-related PVS morphology as these scores were not significantly associated with age (Fig. 7B; $r=-.01, p=.72$ ). After controlling for age, sex, and scanner type, PVS VF in neither the white matter $(t(1391)=-$ $1.09, p=.27)$ nor $B G(t(1391)=-.08, p=.93)$ were significantly associated with age-adjusted standardized working memory scores (Fig. 7C-D). In order to determine the relationship between age-related PVS alterations and working memory performance, we tested for the interaction of 
working memory performance and age where subjects were stratified into high-performing (ageadjusted standardized scores > 100) and low-performing (age-adjusted standardized scores $<100)$. A significant interaction was not observed in the prediction of white matter PVS VF $(F(1,910)=.41, p=.52)$ or BG PVS VF $(F(1,910)=1.07, p=.30)$ after controlling for covariates (Fig. 7E-F).

\section{PVS morphological associations}

White matter regions with high PVS VF, mean diameter, mean solidity and count also tended to be high in the BG (Fig. 8). PVS morphological features were significantly correlated with one another in the BG (SI Appendix, Fig. S4) and subcortical white matter (SI Appendix, Fig. S5), where PVS VF was associated with increased mean diameter and count and decreased mean solidity. In order to better understand the contribution of PVS morphological features to alterations in the overall volume of the PVS, multiple linear regression was carried out on standardized variables to predict the PVS VF from PVS count, diameter and solidity. Within the BG, PVS count, mean diameter and mean solidity collectively account for $82 \%$ of the variance in PVS VF $\left(F(3,1392)=2098, p<.0001\right.$, adjusted $\left.R^{2}=.819\right)$. Mean PVS diameter has the highest predictive power $(\beta=.549, t(1392)=35.49, \quad p<.0001)$, followed by PVS count $(\beta=.449$, $t(1392)=31.76, p<.0001)$, and mean PVS solidity $(\beta=.128, t(1392)=7.06, p<.0001)$. PVS mean diameter and PVS count explain $16 \%$ and $13 \%$ of the unique variance in PVS VF, respectively, while mean PVS solidity explains $1 \%$ of the unique variance in PVS VF. Relative importance analysis was used to understand the extent to which each PVS morphological feature drives the prediction of PVS volume in the multiple regression model. Mean PVS diameter contributes .327 $(95 \% \mathrm{Cl}=[.295, .358])$ to the total proportion of variance, while PVS count and mean solidity contribute $.251[.230, .276]$ and $.241[.225, .257]$, respectively.

Within the white matter, PVS count, mean diameter and mean solidity account for $84 \%$ of the variance in PVS VF $\left(F(3,1392)=2424, p<.0001\right.$, adjusted $\left.R^{2}=.839\right)$. Mean PVS diameter has the highest standardized beta in multiple regression $(\beta=.498, t(1392)=42.76, p<.0001)$, followed by count $(\beta=.377, t(1392)=19.21, p<.0001)$, and mean solidity $(\beta=.285, t(1392)=15.20, p<.0001)$. Mean PVS diameter explains $21.12 \%$ of the unique variance in PVS VF, while PVS count and mean solidity account for $4 \%$ and $3 \%$, respectively. Relative importance analysis shows mean PVS diameter contributes $.315(95 \% \mathrm{Cl}=[.288, .340])$ to the total proportion of variance, while PVS count and mean PVS solidity contribute .301 [.284, .319] and .224 [.206, .240], respectively.

\section{Discussion}

The present study is the first, to our knowledge, to characterize PVS structure across the lifespan utilizing morphological features derived from automated segmentations in the BG and white matter. We found PVS visibility increases with age and was accompanied by increased PVS volume fraction, count and caliber and decreased solidity. Furthermore, PVS enlargement in the $B G$ and white matter followed different age-related trajectories. Within the white matter, temporal regions show the largest percent change in PVS volumetric expansion, while cingulate and occipital regions show the least change. Overall, we observed a significant relationship between PVS burden in childhood and the rate of PVS expansion, where regions characterized by low PVS burden in childhood tended to show the most drastic changes with age. Persistent sex differences in PVS morphology were also observed across the lifespan and differing rates of agerelated PVS alterations were observed in males and females. Our results demonstrate PVS 
enlargement is a dynamic and heterogeneous process that is a feature of healthy aging in cognitively normal subjects across the lifespan.

Our findings of increased PVS volume fraction with age across the lifespan are corroborated by previous studies that have found PVS visibility is apparent in children and adolescents $(17,37,38)$ and increases with age in cognitively normal young adults $(18,39)$ and elderly subjects $(15,29-34)$. Furthermore, or finding of increased PVS count and caliber across the lifespan suggests the observed expansion of the PVS VF is likely attributed to widening of the PVS, and not due to decreased tissue volume associated with age-related brain atrophy. Because working memory performance was not significantly associated with PVS morphology in our dataset, the observed PVS alterations likely reflect normal age-related variation and not neuropathology. Previous research has suggested a strong interdependence between PVS structure and waste clearance efficiency in the brain. Physical alterations that disrupt bulk flow, such as changes to CSF pressure and periarterial blockages, can result in widening of the PVS (40). Previous studies have also shown aging is associated with reduced metabolic waste clearance, which may be attributed to age-related reduction in CSF production (41), decline in arterial pulsatility (42), increase in blood-brain barrier permeability (43) and deposition of insoluble $A B$ aggregates (44). Therefore, age-related PVS enlargement may reflect reduced fluid exchange and elimination from the brain. Because the highest risk factor for the development of neurodegenerative diseases is aging, PVS enlargement and subsequent failure of the waste clearance system may contribute to the accumulation of protein aggregates and render the brain more vulnerable to neurodegenerative pathology or escalate the progression of cognitive dysfunction.

While PVS alterations were observed globally across the lifespan, some noticeable differences between the BG and white matter age-related PVS morphological trajectories. Accelerated enlargement of PVS VF in the BG was predominantly observed in older subjects and coincided with wider diameters and, to a lesser extent, a larger number of visible PVS. Conversely, PVS VF in white matter increased linearly with age across the lifespan and was mainly attributed to increased PVS caliber. The differing PVS trajectories in the white matter and BG may be attributed to differing anatomical characteristics that define vasculature in these structures. PVS in the BG are surrounded by 2 layers of leptomeningeal membranes that allow for direct communication with the subarachnoid space, while PVS in white matter are lined by a single layer of leptomeninges aligned closely with the vessel wall that allow for fluid entry through the subpial space (45). These differences in structural organization and fluid sources may reflect relative efficiencies in the drainage of ISF at different sites in the brain. Another potential explanation for the differing age-related trajectories may be attributed to differences in the cerebral arteries that feed into the network of perforating blood vessels in each structure. White matter PVS envelop arteries that originate from several different major cerebral arteries (46), while BG PVS surround lenticulostriate arteries that branch from the M1 segment of the middle cerebral artery (MCA) (47). The M1 segment of the MCA is the most pathologically affected territory in the brain $(48,49)$ and previous studies have shown the PVS surrounding BG vasculature that stems from the MCA are particularly susceptible to vascular pathology $(11,20-23)$. Because the risk for cerebrovascular disease increases with advancing age (50), the accelerated BG PVS enlargement in older subjects may be due, in part, to pathological vascular influences in the aging cohort.

Within white matter, PVS envelop arteries that originate from cerebral arteries that perforate the convexity cortex (51). The anterior cerebral artery (ACA) supplies blood vessels to 
anterior-frontal regions around the interhemispheric fissure, the MCA supplies parietal, temporal and inferior frontal regions via the M4 cortical segment and the posterior cerebral artery (PCA) supplies occipital regions (46). PVS were not uniformly distributed across the white matter, as we found frontal, parietal and cingulate regions had the highest PVS burden, while occipital and temporal regions have the lowest PVS burden bilaterally. Our findings are in line with previous studies that show the highest PVS burden in frontal and parietal lobes across all age groups (17, $18,29,51)$. Additionally, a recent study in young adults found PVS VF in regions supplied by the ACA and MCA is elevated compared to those supplied by the PCA (52). Overall, we observed regionally-dependent patterns of PVS enlargement trajectories across the lifespan, where PVS levels in childhood were inversely correlated with the rate of growth. Regions fed by the ACA, such as cingulate and prefrontal white matter, have elevated PVS VF in childhood that remains relatively constant across the lifespan, while temporal, inferior frontal and lateral occipital regions supplied by the MCA have low PVS VF in childhood and undergo rapid enlargement across the lifespan. Medial occipital regions supplied by the PCA are an exception to this trend, as PVS VF levels remain low across the lifespan. Together, our findings suggest the mechanisms that contribute to age-related PVS enlargement differ according to the cerebral arteries that support each region.

It is unclear if the age-related increase in PVS cross-section diameter reflects pathological enlargement. From childhood to advancing age, the mean PVS diameter in the present study ranges from $1.85 \mathrm{~mm}$ to $2.36 \mathrm{~mm}$ in the white matter and $1.32 \mathrm{~mm}$ to $1.66 \mathrm{~mm}$ in the basal ganglia. Previous MRI studies acquired at 3T have similarly found the majority of PVS have a maximum diameter of $3 \mathrm{~mm}(7,53)$; however, it is likely that many more PVS go undetected in the brain due to limitations in the spatial resolution afforded by clinical MRI scanners. PVS with submillimeter diameters have been observed with ultra-high field MRI (54), which provides significantly provide visualization of small PVS, and histological studies in vascular tissue suggest the majority of PVS diameters range from .13 to .96 mm (55). Due to the preponderance of smallcaliber PVS in the brain that are undetectable at the current resolution, it is feasible that the observed PVS diameter enlargement may be attributed to the simultaneous expansion of multiple neighboring PVS, thus resulting in the appearance of a single larger PVS (54).

Our finding of increased PVS count with age corroborates previous studies $(51,52)$ and is likely attributed to increased visibility of pre-existing PVS, as opposed to the formation of new ones, because PVS spatially localize with penetrating arterioles and venules that remain static across age. Consistent with our findings of a strong correlation between PVS count and mean diameter, increased PVS count could therefore reflect increased caliber of small PVS previously undetectable with MRI. The age-related increase in PVS count may also be attributed to discontinuities in PVS visibility, where a single PVS is delineated as multiple disconnected segments. While discontinuities may be attributed to partial volume effects in small PVS, others have suggested PVS enlargement can manifest as focal dilations (56) that may be visualized as several separate segments. This latter interpretation is supported by our finding of age-related reductions in PVS solidity, a measure that describes shape complexity based on the fraction of voxels enclosed within the smallest complex polygon, where low solidity tend to describe highly tortuous paths (57). Previous studies have similarly found increased arterial tortuosity with aging (58-60). It is unclear whether the increased complexity of the PVS observed in the present study is due to alterations to the PVS itself, through focal dilations and degradation, or if it is guided by the underlying structure of the vasculature. Together, our finding of increased PVS VF, count and diameter accompanied by decreased solidity across the lifespan point towards age-related enlargement and possibly degradation of PVS. 
Previous studies have shown PVS volumes are larger in males compared to females in adult $(18,32)$ and aging subjects $(24)$. While we found PVS VF was significantly larger in males compared to females in the white matter and BG, males also had significantly more PVS compared to females in the BG, specifically. Androgen and estrogen receptors on vascular endothelial cells act as direct targets for circulating sex hormones $(61,62)$ and the relative distributions of these hormones across the lifespan may contribute to the observed PVS sex differences. While androgens have been shown to protect the blood-brain barrier (63), they can also adversely affect vasculature through neuroinflammatory stimulation (64) and vasoconstriction (65). Therefore, the unique actions of circulating androgens on the cerebrovascular system may further exacerbate age-related PVS enlargement in males. We also found age-related increases to the volume and diameter of white matter PVS occur more rapidly in females compared to males, where PVS burden in females is lower in childhood and adulthood and equalizes with males in older subjects. Estrogen is atheroprotective and has been shown to suppress the cerebrovascular inflammatory response $(66,67)$, reduce blood-brain barrier permeability (68) and induce vasodilation (69). However, the protective effects of estradiol wanes with age as the gonadal production of sex hormones declines after menopause (70) and is reflected by an age-related increase in blood-brain barrier permeability in females compared to males (71). Therefore, the accelerated PVS enlargement in females compared to males across the lifespan may be attributed to age-related reductions in circulating estrogen levels.

While the current study utilizes a granular approach to explore the spatial distribution of PVS morphological characteristics across age, several limitations should be considered. HCP is a vast dataset acquired on different scanners at multiple sites and data for the developing and aging cohorts were acquired with slightly different MRI acquisitions than the young adult cohort. To overcome this, we employed robust post-processing harmonization techniques to account for differences in acquisitions and controlled for scanner type as a covariate of no interest in our analyses. Furthermore, we analyzed cohorts separately and found these results were consistent with our lifespan trends. Another limitation was the cross-sectional nature of our study and associations between PVS morphology and age point towards differences, not within-subject changes, across the lifespan. Our research is also limited by the spatial resolution afforded with clinically feasible MRI. In a recent simulation study, researchers found structural MRI data can accurately quantify PVS with diameters greater than twice the image resolution (51). Therefore, the presence of small PVS may lead to a systemic over-estimation of PVS diameters.

The present study sought to characterize the time course of PVS morphological alterations in the BG and subcortical white matter regions across the lifespan in a large cohort of $\sim 1400$ cognitively normal subjects. We found PVS within white matter regions and the BG undergo distinct age-related trajectories that may be indicative of their respective roles in pathological conditions characterized by failure of the waste clearance system. Furthermore, the rate of PVS enlargement in white matter regions were dependent on the degree of PVS burden in childhood, highlighting a critical link between waste clearance processes in childhood and in aging. The findings presented here will aid in our understanding of the role of PVS in health and disease by providing a benchmark from which pathological PVS alterations can be compared. Therefore, future studies should aim to understand the pathophysiological and normative mechanisms that give rise to PVS enlargement in order to better assess its utility as a diagnostic biomarker for neurodegenerative and cerebrovascular disease. 


\section{Materials and Methods}

\section{Study Participants}

Cognitively normal subjects from childhood through advanced age were recruited and scanned through the Washington University-University of Minnesota (WashU-Minn) Human Connectome Project and the Lifespan Human Connectome Project in Development and Aging. Structural neuroimaging and demographic information from 1394 participants (784 females) across the lifespan were included in the present study $(M \pm S D=34.17 \pm 20.07$ years, 8.08 - 89.75 years). Across studies, efforts were made to recruit participants that were representative of the racial and ethnic composition of the United States population.

HCP Development (HCP-D) The sample of typically developing children and adolescents used in the present study were derived from the Lifespan Human Connectome Project in Development (72). Subjects were recruited and scanned in Boston, Los Angeles, Minneapolis and St. Louis. Participants were excluded if they were born premature, require special educational services, had MRI contraindications, or had a history of serious medical problems, head injury, endocrine disorders, psychiatric disorders, or neurodevelopmental disorders. The goal of the HCP-D was to enroll at least 1,300 participants; however, 655 subjects (332 female) with neuroimaging data were available at the time of this study. Of the participants originally considered, 140 subjects were excluded in the HCP-D 2.0 release of the data, 32 were excluded due to poor structural MRI data quality, 6 subjects were excluded due to processing pipeline failures, and 1 subject was excluded due to anatomical abnormalities, resulting in the inclusion of 471 subjects ( 260 female) from the HCP-D cohort in the present analyses $(M \pm S D=14.36 \pm 3.77$ years, $8.08-21.92$ years).

HCP Young Adults (HCP-YA) The sample of healthy young adults were acquired through the WUMinn Human Connectome Project at Washington University in St. Louis (73). HCP-YA utilized the same exclusionary criteria as the HCP-D; HCP-YA also excluded subjects with scores below 25 on the mini-mental state exam (MMSE). The study aimed to recruit 1,200 subjects; however, 1112 subjects with neuroimaging data were available at the time of the present study. Of those considered, 51 subjects were excluded due to poor structural data quality, 643 subjects were randomly excluded due to familial relationships with other participants, 12 subjects were excluded due to processing pipeline failures, and 1 subject was excluded due to anatomical abnormalities, resulting in a total of 405 subjects from the HCP-YA cohort (226 females) used in the present analyses $(M \pm S D=28.73 \pm 3.78$ years, $22.00-37.00$ years $)$.

HCP Aging (HCP-A) The sample of cognitively normal aging adults older than 36 years of age were acquired through the Lifespan Human Connectome Project in Aging (74). Participants were recruited and scanned at Washington University St Louis, University of Minnesota, Massachusetts General Hospital and the University of California Los Angeles. In addition to the exclusionary criteria listed for HCP-D and HCP-YA, individuals with impaired cognitive abilities were excluded from HCP-A according to tiered age-appropriate cut-off scores. While over 1,200 subjects will ultimately be enrolled in the study, there were 687 subjects with neuroimaging data available at the time of analysis. Of those originally considered, 105 subjects were excluded following the 2.0 release of the HCP-A dataset, 43 were additionally excluded due to poor structural data quality following quality assurance purposes, 18 subjects were excluded due to processing pipeline failures, and 1 subject was excluded due to anatomical abnormalities, resulting in the inclusion of a total of 520 subjects (298 females) from the HCP-A cohort in the present analyses $(M \pm S D=56.35 \pm 13.65$ years, $36.00-89.75$ years $)$. 


\section{MRI Acquisition}

HCP-YA Participants were scanned on a Siemens 3T Connectome Skyra with a $100 \mathrm{mT}$ gradient coil and a 32-channel Siemens receive head coil (75). B0 field maps, B1- and B1+ maps were collected to correct for readout distortion and intensity inhomogeneities. T1-weighted (T1w) images were collected as a 3D single-echo magnetization prepared - rapid gradient echo (MPRAGE) images with the following acquisition parameters: voxel size $=.7 \mathrm{~mm}$ isotropic, $\mathrm{FOV}=224$ $\mathrm{mm}$, matrix $=320,256$ sagittal slices per slab, $\mathrm{TR}=2400 \mathrm{~ms}, \mathrm{TE}=2.14 \mathrm{~ms}, \mathrm{TI}=1000 \mathrm{~ms}, \mathrm{FA}=$ 8 degrees, Bandwidth $=210 \mathrm{~Hz}$ per pixel, echo spacing $=7.6 \mathrm{~ms}$, GRAPPA factor $=2,10 \%$ phase encoding oversampling (A-P), dwell time $=7.4 \mu \mathrm{s}$. T2-weighted (T2w) images were collected as 2 variable flip angle turbo spin-echo sequences averaged together with the following acquisition parameters: voxel size $=.7 \mathrm{~mm}$ isotropic, FOV $=224 \mathrm{~mm}$, matrix $=320,256$ sagittal slices per slab, TR $=3200 \mathrm{~ms}, \mathrm{TE}=565 \mathrm{~ms}, \mathrm{BW}=744 \mathrm{~Hz} /$ pixel, no fat suppression pulse, GRAPPA $=2$, turbo factor $=314$, echo train length $=1105$ echoes, $10 \%$ phase encoding oversampling, dwell time $=2.1 \mu \mathrm{s}$.

HCP-D and HCP-A Slight variations in the acquisition parameters were made for the HCP-A and HCP-D cohorts to accommodate the unique challenges of working with young and elderly populations (76). HCP-D and HCP-A participants were scanned on a variant of the HCP-YA Connectome scanner, the Siemens 3T Prisma with an $80 \mathrm{mT}$ gradient coil and a Siemens 32channel prisma head coil. T1w multi-echo MP-RAGE scans were acquired with the following acquisition parameters: voxel size $=.8 \mathrm{~mm}$ isotropic, 4 echoes per line of k-space, $\mathrm{FOV}=256 \mathrm{x}$ $240 \times 166 \mathrm{~mm}$, matrix $=320 \times 300 \times 208$ slices, $7.7 \%$ slice oversampling, GRAPPA = 2, pixel bandwidth $=744 \mathrm{~Hz} /$ pixel, $\mathrm{TR}=2500 \mathrm{~ms}, \mathrm{TI}=1000 \mathrm{~ms}, \mathrm{TE}=1.8 / 3.6 / 5.4 / 7.2 \mathrm{~ms}, \mathrm{FA}=8$ degrees. Motion-induced re-acquisition were allowed for up to 30 TRs. T2w turbo spin echo (TSE) scans were collected from each subject with the following acquisition parameters: voxel size $=.8 \mathrm{~mm}$ isotropic, 4 echoes per line of k-space, FOV $=256 \times 240 \times 166 \mathrm{~mm}$, matrix $=320 \times 300 \times 208$ slices, $7.7 \%$ slice oversampling, GRAPPA $=2$, pixel bandwidth $=744 \mathrm{~Hz} /$ pixel, $\mathrm{TR}=3200 \mathrm{~ms}$, TE $=564 \mathrm{~ms}$, turbo factor $=314$. Motion-induced re-acquisition were allowed up to $25 \mathrm{TRs}$.

\section{Quality assurance procedures}

Prior to data processing and PVS segmentation, all T1w and T2w images were visually inspected and scans with poor data quality were excluded from analyses. Poor scan quality was characterized by T1w, T2w or EPC volumes with excessive head motion, reduced GM/WM tissue contrast, unidentifiable anatomy, blurred images and salt-and-pepper noise, which obstructed the visibility of PVS. Additionally, EPC images with contrast inhomogeneities due to slight variations in T1w and T2w data quality or registration failures were excluded.

\section{MRI data preprocessing}

All T1w and T2w images were preprocessed using the HCP minimal processing pipeline version 4.0.1 (75) in parallel with the LONI pipeline (77) . HCP-A and HCP-D structural images were resampled to a spatial resolution of $.7 \mathrm{~mm}$ isotropic to match that of HCP-YA. Following gradient distortion correction, T2w images were aligned to T1w images using rigid body registration and then images were transformed to $\mathrm{MNI}$ space and brought into alignment with the anterior and posterior commissure (ACPC). Brain extraction and readout distortion correction using a field map were then carried out. T2w images were registered to subject T1w native space using boundary- 
based registration (BBR) with 6 degrees of freedom (78). T1w and T2w images were corrected for B1- and B1 + bias and then registered to MNI space with an affine transformation implemented with FLIRT followed by a nonlinear registration implemented with FNIRT.

\section{Subcortical segmentation}

In order to characterize regional subcortical PVS features, T1w images were processed with Freesurfer version 6 (http://surfer.nmr.mgh.harvard.edu/). Images were first downsampled to a voxel size of $1 \mathrm{~mm}$ isotropic to accommodate spatial resolution limitations of Freesurfer (75). Data processing included motion correction, intensity normalization, removal of non-brain tissue and automated Talairach transformation (79-81). Subcortical white matter and BG were segmented using an atlas-based approach $(82,83)$. Subcortical white matter was further parcellated into 68 regional volumes (34 regions per hemisphere) labeled according to the nearest cortical label (82, 83) defined by the Desikan-Killiany atlas (84).

\section{Perivascular space segmentation}

PVS were automatically segmented and quantified using the processing technique described in (35). Adaptive non-local mean filtering using image self-similarity properties was applied to the co-registered and bias field-corrected T1w and T2w images (85). In order to increase visibility of the PVS in the subcortical white matter and BG for segmentation, an enhanced PVS contrast (EPC) was then generated by dividing the T1w image by the T2w image (35).

Frangi filters were applied to the EPC and T2w images (86) using the Quantitative Imaging Toolkit (QIT) (87) to create PVS vesselness maps, which describes the extent to which a voxel corresponds to the tubular structure of the PVS. Frangi filter parameters were set to default values of $\alpha=\beta=0.5$ and $c$ was set to half the maximum Hessian norm according to (86). The vesselness scale ranged from 0.1 to 5 voxels. Binary PVS maps were generated by thresholding the vesselness maps using a value optimized for the highest concordance and correlation with PVS counts from expert readers (EPC: scaled $t=1.5$; T2w: scaled $t=2.7$ ). The CSF volumetric mask generated by Freesurfer was dilated using a spherical kernel with a $1.4 \mathrm{~mm}$ radius and used to exclude voxels at the interface between white matter and ventricles to avoid incorrect classification of outside tissue voxels.

White matter hyperintensities (WMH) are anomalous structural features indicative of tissue pathology (88). WMH is more common in the normal aging brain compared to younger brains (89) and has a similar contrast to PVS. While vesselness thresholding excludes most large WMH from consideration during PVS segmentation, since WMH can be incorrectly classified as PVS. The influence of WMH on PVS segmentation was assessed in a subset of 200 participants (100 HCP. A, 50 HCP-YA, and 50 HCP-D subjects), where PVS maps were visually inspected and manually edited to remove segmentation false positives by a group of 4 trained raters, including $\mathrm{WMH}$, ventricular walls due to inaccurate tissue segmentation and other erroneously labeled regions with hyper-intense EPC contrast. To ensure consistency among raters, 33\% of the subjects were inspected by more than one rater. The corrected and uncorrected PVS maps were compared to determine if manual correction significantly improves analyses. Out of the 200 subjects randomly selected for analysis, 10 (5\%) (1 HCP-D, 1 HCP-YA and 8 HCP-A) were noted to have moderate or severe $\mathrm{WMH}$ according to a visual rating scale ranging from 1 to 3 based on the number and size of $\mathrm{WMH}(1=$ no $\mathrm{WMH}, 3=$ several severe $\mathrm{WMH})$. Overall, the false positive rate across all cohorts was $4.5 \%$ (HCP-D: $4.1 \%$, HCP-YA: $7.3 \%$, HCP-A: $3.1 \%$ ). WMH severity was not 
significantly correlated with the PVS segmentation false positive rate $(b=.014, t(198)=1.65$, $p=.10)$. Therefore, it is unlikely that PVS structural alterations in the present study are not attributed to the presence of WMH in the PVS segmentation method.

\section{Perivascular space feature extraction}

The PVS volume fraction was defined as fraction of the volume of space occupied by PVS and was calculated by dividing the PVS volume by the subcortical white matter and basal ganglia volume. The PVS morphological properties of count, mean diameter and mean solidity were extracted from the 3-D volumetric mask of PVS using the Matlab function regionprops3. The PVS count summed up the number of contiguous PVS-labeled voxels within a given region of interest. PVS diameter reflects the average cross-sectional diameter and is calculated using the mean of the second and third principal eigenvectors of an ellipsoid constructed with the same normalized second central moments as the segmented PVS. The PVS solidity corresponds to the proportion of the PVS-labeled voxels within the convex hull that contains a given PVS segment. Because segmented PVS are not always cluster of voxels, The PVS mean solidity was calculated on PVS with a length of 5 contiguous voxels or greater. For analyses, the mean PVS solidity and PVS diameter are provided for each white matter and BG region of interest.

\section{Working memory performance}

Several cognitive and psychological assessments were administered to subjects in the HCP-D (72), HCP-YA (90) and HCP-A datasets (74). To assess working memory performance, subjects were administered the NIH Toolbox List Sorting Task (91). In the task, a sequence of familiar stimuli (food or animals) are visually and orally presented to subjects, who are then required to name the series of objects in order of size from smallest to largest. This task has been validated in subjects between 3 and 85 years of age and is significantly correlated with others measures of executive function (92). Age-adjusted standardized scores were computed based on different age groups (ages 3-11 years, 12-59 years, and >60 years); therefore, analyses in the present study were performed within a subset of participants that comprises 919 subjects (520 females) between 12 and 59 years of age (30.97 \pm 12.41 years).

\section{Statistical analysis}

Harmonization Procedure To characterize age-related PVS alterations across the lifespan, analyses were the carried out on the combined HCP datasets. In order to adjust for non-biological variance inherent in multi-site research studies, such as differences in study sites and acquisition protocols, we harmonized the combined lifespan datasets using ComBat-GAM (https://github.co $\mathrm{m} / \mathrm{rpomponio}$ /neuroHarmonize). ComBat-GAM is an extension of the batch effect correction tool ComBat commonly used in genomics and recently developed to account for multi-site scanner effects. It has been previously shown that ComBat removes unwanted scanner effects, while preserving biological associations, in a large multi-study analysis that investigated cortical thickness in health and disease (93). ComBat-GAM additionally utilizes an generalized additive model (GAM) with a penalized non-linear term to model the dynamic age effects typically observed across the lifespan (94). ComBat-GAM was applied to the PVS metrics extracted from the $B G$ and white matter regions to correct for HCP cohort effects while preserving the effects of sex and the nonlinear effects of age. The harmonization procedure was also run within an empirical Bayes framework, which assumes features share the same common distribution. 
Cohort-specific analyses Statistical analysis were carried out with $\mathrm{R}$ version 3.1 .2 and were assessed separately for each cohort. To assess the influence of age on PVS morphometric features (mean diameter, mean solidity, count and VF) within the subcortical white matter and basal ganglia, a general linear model (stats v.3.6.2) was fit for each cohort with the form: PVS = $B_{0}+B_{1}{ }^{*}$ age $+B_{2}{ }^{*}$ sex $+B_{3}{ }^{*}$ scanner $+B_{4}{ }^{*}$ volume, where the dependent variable reflects the morphological feature for a given structure and sex, scanner type and white matter of BG volume are considered covariates of no interest. The main effect of sex after accounting for age and scanner type were assessed with analysis of covariance (ANCOVA). Statistical tests were corrected for multiple comparisons using a Bonferroni-adjusted significance threshold of $p<.002$ $(.05 / 24)$.

Combined cohort analyses Combined analyses utilized the harmonized data and included HCP. D, HCP-YA and HCP-A to assess PVS morphology across the lifespan. The main effect of sex and age*interaction on PVS features after controlling for covariates was assessed with ANCOVA. For age effects, Bayesian information criteria (BIC) was used to identify the model that accounts for the most age-related variance in PVS morphology among the following 4 models typically used to characterize lifespan trajectories: (1) linear regression, (2) second-order polynomial model, (3) Poisson curve, and (4) nonlinear growth curve. For the PVS features within the total white matter and $B G$, the best fit models were either linear regression (PVS $=B_{0}+B_{1}{ }^{*}$ age) or the second-order polynomial model (PVS $=B_{0}+B_{1}{ }^{*}$ age $+B_{2}{ }^{*} a^{2}{ }^{2}$ ). A nonlinear growth curve was fit for each parcellated white matter region to explain the relationship between PVS VF and age in order to compare the rate of PVS VF change among regions. The model was of the form: PVS $=a e^{k^{*} \text { age }}$, where $k$ reflects the growth rate. The $95 \%$ confidence interval for each growth model coefficient and predicted age at 8 years of age were generated with bootstrap resampling with replacement $(N=10,000)$. Statistical tests were corrected for multiple comparisons using a Bonferroni-adjusted significance threshold of $p<.006(.05 / 8)$.

\section{Acknowledgments}

The image computing resources provided by the Laboratory of Neuro Imaging Resource (LONIR) at USC are supported in part by National Institutes of Health (NIH) National Institute of Biomedical Imaging and Bioengineering (NIBIB) grant P41EB015922. Author KML is supported by the National Institute on Aging (NIA) of the NIH Institutional Training Grant T32AG058507. The research reported in this publication was supported by the National Institute of Mental Health $(\mathrm{NIMH})$ of the NIH under the award number RF1MH123223, the NIA of the NIH under the award number R01-AG070825, and the USC ADRC 1P30AG066530-01.

Author contributions: Kirsten M. Lynch: Conceptualization, Methodology, Formal analysis, Writing - Original Draft, Writing - Review and Editing, Visualization; Farshid Sepehrband: Conceptualization, Methodology, Software, Writing - Review \& Editing, Resources, Data Curation, Supervision; Arthur W. Toga: Resources, Writing - Review \& Editing, Supervision, Funding acquisition; Jeiran Choupan: Resources, Data Curation, Software, Writing - Review \& Editing, Supervision, Project administration, Funding acquisition

Competing Interest Statement: The perivascular space mapping technology is part of a pending patent owned by FS and JC, with no financial interest/conflict.

\section{References}

1. J. M. Wardlaw, et al., Perivascular spaces in the brain: anatomy, physiology and pathology. Nat. Rev. Neurol. 16, 137-153 (2020). 
2. B. Bedussi, M. Almasian, J. De Vos, E. Vanbavel, E. N. T. P. Bakker, Paravascular spaces at the brain surface: Low resistance pathways for cerebrospinal fluid flow. J. Cereb. Blood Flow Metab. 38, 719-726 (2018).

3. A. Bacyinski, M. Xu, W. Wang, J. Hu, The Paravascular Pathway for Brain Waste Clearance: Current Understanding, Significance and Controversy. Front. Neuroanat. 11, 1-8 (2017).

4. J. J. lliff, et al., A paravascular pathway facilitates CSF flow through the brain parenchyma and the clearance of interstitial solutes, including amyloid $\beta$. Sci. Transl. Med. 4 (2012).

5. J. J. lliff, et al., Cerebral arterial pulsation drives paravascular CSF-interstitial fluid exchange in the murine brain. J. Neurosci. 33, 18190-18199 (2013).

6. A. Keable, et al., Deposition of amyloid $\beta$ in the walls of human leptomeningeal arteries in relation to perivascular drainage pathways in cerebral amyloid angiopathy. Biochim. Biophys. Acta - Mol. Basis Dis. 1862, 1037-1046 (2016).

7. F. Troili, et al., Perivascular Unit: This Must Be the Place. The Anatomical Crossroad Between the Immune, Vascular and Nervous System. Front. Neuroanat. 14, 1-17 (2020).

8. M. D. Sweeney, K. Kisler, A. Montagne, A. W. Toga, B. V. Zlokovic, The role of brain vasculature in neurodegenerative disorders. Nat. Neurosci. 21, 1318-1331 (2018).

9. M. Cavallari, et al., Evaluating the Association between Enlarged Perivascular Spaces and Disease Worsening in Multiple Sclerosis. J. Neuroimaging 28, 273-277 (2018).

10. R. A. Opel, et al., Effects of traumatic brain injury on sleep and enlarged perivascular spaces. J. Cereb. Blood Flow Metab. (2018) https:/doi.org/10.1177/0271678X18791632.

11. A. Charidimou, et al., Enlarged perivascular spaces as a marker of underlying arteriopathy in intracerebral haemorrhage: A multicentre MRI cohort study. J. Neurol. Neurosurg. Psychiatry 84, 624-629 (2013).

12. G. Banerjee, et al., MRI-visible perivascular space location is associated with Alzheimer's disease independently of amyloid burden. Brain 140, 1107-1116 (2017).

13. F. Sepehrband, et al., Volumetric distribution of perivascular space in relation to mild cognitive impairment. Neurobiol. Aging 99, 28-43 (2021).

14. E. K. Donahue, et al., Global and Regional Changes in Perivascular Space in Idiopathic and Familial Parkinson's Disease. Mov. Disord., 1-12 (2021).

15. Y. C. Zhu, et al., Frequency and location of dilated Virchow-Robin spaces in elderly people: A population-based 3D MR imaging study. Am. J. Neuroradiol. 32, 709-713 (2011).

16. A. Laveskog, R. Wang, L. Bronge, L.-O. Wahlund, C. Qiu, Perivascular Spaces in Old Age: Assessment, Distribution, and Correlation with White Matter Hyperintensities. Am. J. Neuroradiol. 39, 70 LP - 76 (2018).

17. J. Piantino, et al., Characterization of MR imaging-visible perivascular spaces in the white matter of healthy adolescents at 3T. Am. J. Neuroradiol. 41, 2139-2145 (2020).

18. G. Barisano, N. Sheikh-Bahaei, M. Law, A. W. Toga, F. Sepehrband, Body mass index, time of day, and genetics affect perivascular spaces in the white matter. J. Cereb. Blood Flow Metab. (2020) https:/doi.org/10.1177/0271678X20972856.

19. Y. Hou, et al., Ageing as a risk factor for neurodegenerative disease. Nat. Rev. Neurol. 15, 565-581 (2019).

20. G. M. Potter, et al., Enlarged perivascular spaces and cerebral small vessel disease. Int. J. Stroke 10, 376-381 (2015).

21. F. N. Doubal, A. M. J. MacLullich, K. J. Ferguson, M. S. Dennis, J. M. Wardlaw, Enlarged Perivascular Spaces on MRI Are a Feature of Cerebral Small Vessel Disease. Stroke 41, 450-454 (2010).

22. S. Martinez-Ramirez, et al., Topography of dilated perivascular spaces in subjects from a memory clinic cohort. Neurology 80, 1551-1556 (2013).

23. A. Charidimou, et al., MRI-visible perivascular spaces in cerebral amyloid angiopathy and hypertensive arteriopathy. Am. Acad. Neurol. 88, 1157-64 (2017).

24. J. Ramirez, et al., Visible Virchow-Robin spaces on magnetic resonance imaging of Alzheimer's disease patients and normal elderly from the Sunnybrook dementia study. J. Alzheimer's Dis. 43, 415-424 (2015).

25. G. Banerjee, et al., MRI-visible perivascular space location is associated with Alzheimer's disease 
independently of amyloid burden. Brain 140, 1107-16 (2017).

26. A. Charidimou, et al., White Matter Perivascular Spaces on Magnetic Resonance Imaging - Marker of Cerebrovascular Amyloid Burden? Stroke 46, 1707-1709 (2015).

27. M. Etemadifar, et al., Features of Virchow-Robin spaces in newly diagnosed multiple sclerosis patients. Eur. J. Radiol. 80, 104-108 (2011).

28. I. D. Kilsdonk, et al., Perivascular spaces in MS patients at 7 Tesla MRI: A marker of neurodegeneration? Mult. Scler. J. 21, 155-162 (2015).

29. A. Laveskog, R. Wang, L. Bronge, L. O. Wahlund, C. Quu, Perivascular spaces in old age: Assessment, distribution, and correlation with white matter hyperintensities. Am. J. Neuroradiol. 39, 70-76 (2018).

30. F. Francis, L. Ballerini, J. M. Wardlaw, Perivascular spaces and their associations with risk factors, clinical disorders and neuroimaging features: A systematic review and meta-analysis. Int. J. Stroke 14, 359-371 (2019).

31. Y. Yakushiji, et al., Topography and associations of perivascular spaces in healthy adults; The kashima scan study. Neurology 83, 2116-2123 (2014).

32. Y. C. Zhu, et al., Severity of dilated virchow-robin spaces is associated with age, blood pressure, and MRI markers of small vessel disease: A population-based study. Stroke 41, 2483-2490 (2010).

33. J. Gutierrez, T. Rundek, M. S. V. Ekind, R. L. Sacco, C. B. Wright, Perivascular spaces are associated with atherosclerosis: An insight from the northern manhattan study. Am. J. Neuroradiol. 34, 1711-1716 (2013).

34. P. Huang, et al., Factors Associated With the Dilation of Perivascular Space in Healthy Elderly Subjects. Front. Aging Neurosci. 13, 1-9 (2021).

35. F. Sepehrband, et al., Image processing approaches to enhance perivascular space visibility and quantification using MRI. Sci. Rep. 9, 12351 (2019).

36. A. Cochrane, V. Simmering, C. Shawn Green, Fluid intelligence is related to capacity in memory as well as attention: Evidence from middle childhood and adulthood. PLoS One 14, 1-24 (2019).

37. S. Groeschel, W. K. Chong, R. Surtees, F. Hanefeld, Virchow-Robin spaces on magnetic resonance images: normative data, their dilatation, and a review of the literature. Neuroradiology 48, 745-754 (2006).

38. N. K. Rollins, C. Deline, M. C. Morriss, Prevalence and clinical significance of dilated Virchow-Robin spaces in childhood. Radiology 189, 53-57 (1993).

39. Y. Choi, et al., MRI-visible dilated perivascular spaces in healthy young adults: A twin heritability study. Hum. Brain Mapp. 41, 5313-5324 (2020).

40. N. A. Jessen, A. S. F. Munk, I. Lundgaard, M. Nedergaard, The Glymphatic System - A Beginner's Guide. Neurochem Res 40, 2583-2599 (2015).

41. D. Fleischman, et al., Cerebrospinal Fluid Pressure Decreases with Older Age. PLoS One 7, 1-9 (2012).

42. S. J. Zieman, V. Melenovsky, D. A. Kass, Mechanisms, pathophysiology, and therapy of arterial stiffness. Arterioscler. Thromb. Vasc. Biol. 25, 932-943 (2005).

43. B. T. Kress, et al., Impairment of paravascular clearance pathways in the aging brain. Ann. Neurol. 76, 845-861 (2014).

44. R. O. Weller, M. Subash, S. D. Preston, I. Mazanti, R. O. Carare, Perivascular drainage of amyloid$\beta$ peptides from the brain and its failure in cerebral amyloid angiopathy and Alzheimer's disease. Brain Pathol. 18, 253-266 (2008).

45. H. Pollock, M. Hutchings, R. O. Weller, E. T. Zhang, Perivascular spaces in the basal ganglia of the human brain: Their relationship to lacunes. J. Anat. 191, 337-346 (1997).

46. D. E. Kim, et al., Mapping the Supratentorial Cerebral Arterial Territories Using 1160 Large Artery Infarcts. JAMA Neurol. 76, 72-80 (2019).

47. S. Marinković, H. Gibo, M. Milisavljević, M. Ćetković, Anatomic and clinical correlations of the lenticulostriate arteries. Clin. Anat. 14, 190-195 (2001).

48. Y. S. Ng, J. Stein, M. M. Ning, R. M. Black-Schaffer, Comparison of clinical characteristics and functional outcomes of ischemic stroke in different vascular territories. Stroke 38, 2309-2314 (2007).

49. O. O. Zaidat, et al., Middle Cerebral Artery Aneurysm Endovascular and Surgical Therapies: Comprehensive Literature Review and Local Experience. Neurosurg. Clin. N. Am. 25, 455-469 
(2014).

50. A. J. Hauer, et al., Age-specific vascular risk factor profiles according to stroke subtype. J. Am. Heart Assoc. 6 (2017).

51. W. H. Bouvy, et al., Visualization of perivascular spaces and perforating arteries with 7 T magnetic resonance imaging. Invest. Radiol. 49, 307-313 (2014).

52. X. Zong, et al., Morphology of perivascular spaces and enclosed blood vessels in young to middleaged healthy adults at 7T: Dependences on age, brain region, and breathing gas. Neuroimage 218, 116978 (2020).

53. M. Del C. Valdes Hernandez, R. J. Piper, X. Wang, I. J. Deary, J. M. Wardlaw, Towards the automatic computational assessment of enlarged perivascular spaces on brain magnetic resonance images: A systematic review. J. Magn. Reson. Imaging 38, 774-785 (2013).

54. G. Barisano, M. Law, R. M. Custer, A. W. Toga, F. Sepehrband, Perivascular Space Imaging at Ultrahigh Field MR Imaging. Magn. Reson. Imaging Clin. N. Am. 29, 67-75 (2021).

55. C. Pesce, F. Carli, Allometry of the perivascular spaces of the putamen in aging. Acta Neuropathol. 76, 292-294 (1988).

56. J. M. Wardlaw, et al., Neuroimaging standards for research into small vessel disease and its contribution to ageing and neurodegeneration. Lancet Neurol. 12, 822-838 (2013).

57. L. M. Westrate, J. A. Drocco, K. R. Martin, W. S. Hlavacek, J. P. MacKeigan, Mitochondrial morphological features are associated with fission and fusion events. PLoS One 9 (2014).

58. L. Del Corso, et al., Tortuosity, kinking, and coiling of the carotid artery: Expression of atherosclerosis or aging? Angiology 49, 361-371 (1998).

59. K. S. Cha, M. H. Kim, H. J. Kim, Prevalence and clinical predictors of severe tortuosity of right subclavian artery in patients undergoing transradial coronary angiography. Am. J. Cardiol. 92, 12201222 (2003).

60. R. S. Schnerr, et al., Pulsatility of lenticulostriate arteries assessed by 7 Tesla flow MRIMeasurement, reproducibility, and applicability to aging effect. Front. Physiol. 8, 1-10 (2017).

61. K. L. Zuloaga, S. N. Swift, R. J. Gonzales, T. J. Wu, R. J. Handa, The androgen metabolite, 5aandrostane-3 $\beta, 17 \beta$-diol, decreases cytokine-induced cyclooxygenase-2, vascular cell adhesion molecule-1 expression, and P-glycoprotein expression in male human brain microvascular endothelial cells. Endocrinology 153, 5949-5960 (2012).

62. K. L. Zuloaga, D. T. O'Connor, R. J. Handa, R. J. Gonzales, Estrogen receptor beta dependent attenuation of cytokine-induced cyclooxygenase-2 by androgens in human brain vascular smooth muscle cells and rat mesenteric arteries. Steroids 77, 835-844 (2012).

63. A. Atallah, S. Mhaouty-Kodja, V. Grange-Messent, Chronic depletion of gonadal testosterone leads to blood-brain barrier dysfunction and inflammation in male mice. J. Cereb. Blood Flow Metab. 37, 3161-3175 (2017).

64. R. J. Gonzales, S. P. Duckles, D. N. Krause, Dihydrotestosterone stimulates cerebrovascular inflammation through NFKB, modulating contractile function. J. Cereb. Blood Flow Metab. 29, 244253 (2009).

65. R. J. Gonzales, D. N. Krause, S. P. Duckles, Testosterone suppresses endothelium-dependent dilation of rat middle cerebral arteries. Am. J. Physiol. - Hear. Circ. Physiol. 286, 552-560 (2004).

66. L. Sunday, M. M. Tran, D. N. Krause, S. P. Duckles, Estrogen and progestagens differentially modulate vascular proinflammatory factors. Am. J. Physiol. - Endocrinol. Metab. 291, 261-267 (2006).

67. A. Razmara, et al., Mitochondrial effects of estrogen are mediated by estrogen receptor $\alpha$ in brain endothelial cells. J. Pharmacol. Exp. Ther. 325, 782-790 (2008).

68. M. Burek, P. A. Arias-Loza, N. Roewer, C. Y. Förster, Claudin-5 as a novel estrogen target in vascular endothelium. Arterioscler. Thromb. Vasc. Biol. 30, 298-304 (2010).

69. T. Murata, H. H. Dietrich, C. Xiang, R. G. Dacey, G protein-coupled estrogen receptor agonist improves cerebral microvascular function after hypoxia/reoxygenation injury in male and female rats. Stroke 44, 779-785 (2013).

70. R. P. Wildman, et al., Associations of endogenous sex hormones with the vasculature in menopausal women: The Study of Women's Health Across the Nation (SWAN). Menopause 15, 414-421 (2008). 
71. F. Liu, R. Yuan, S. E. Benashski, L. D. McCullough, Changes in experimental stroke outcome across the life span. J. Cereb. Blood Flow Metab. 29, 792-802 (2009).

72. L. H. Somerville, et al., The Lifespan Human Connectome Project in Development: A large-scale study of brain connectivity development in 5-21 year olds. Neuroimage 183, 456-468 (2018).

73. D. C. Van Essen, et al., The WU-Minn Human Connectome Project: An overview. Neuroimage 80, 62-79 (2013).

74. S. Y. Bookheimer, et al., The Lifespan Human Connectome Project in Aging: An overview. Neuroimage 185, 335-348 (2019).

75. M. F. Glasser, et al., The minimal preprocessing pipelines for the Human Connectome Project. Neuroimage 80, 105-124 (2013).

76. M. Harms, et al., Extending the Human Connectome Project across ages: Imaging protocols for the Lifespan Development and Aging projects. Neuroimage 183, 972-984 (2018).

77. I. D. Dinov, et al., Efficient, distributed and interactive neuroimaging data analysis using the LONI pipeline. Front. Neuroinform. 3 (2009).

78. D. N. Greve, B. Fischl, Accurate and robust brain image alignment using boundary-based registration. Neuroimage 48, 63-72 (2009).

79. M. Reuter, H. D. Rosas, B. Fischl, Highly Accurate Inverse Consistent Registration: A Robust Approach. Neuroimage 53, 1181-1196 (2010).

80. F. Segonne, et al., A hybrid approach to the skull stripping problem in MRI. Neuroimage 22, 10601075 (2004).

81. J. G. Sled, A. P. Zijdenbos, A. C. Evans, A nonparametric method for automatic correction of intensity nonuniformity in MRI data. IEEE Trans. Med. Imaging 17, 87-97 (1998).

82. B. Fischl, et al., Whole brain segmentation: automated labeling of neuroanatomical structures in the human brain. Neuron 33, 341-355 (2002).

83. B. Fischl, et al., Sequence-independent segmentation of magnetic resonance images. Neuroimage 23, S69-S84 (2004).

84. R. S. Desikan, et al., An automated labeling system for subdividing the human cerebral cortex on MRI scans into gyral based regions of interest. Neuroimage 31, 968-980 (2006).

85. J. V Manjón, P. Coupé, L. Martí-Bonmatí, D. L. Collins, M. Robles, Adaptive non-local means denoising of MR images with spatially varying noise levels. J. Magn. Reson. Imaging 31, 192-203 (2010).

86. A. F. Frangi, W. J. Niessen, K. L. Vincken, M. A. Viergever, Multiscale vessel enhancement filtering in International Conference on Medical Image Computing and Computer-Assisted Intervention, (Springer, 1998), pp. 130-137.

87. R. P. Cabeen, D. H. Laidlaw, A. W. Toga, Quantitative imaging toolkit: Software for interactive 3D visualization, data exploration, and computational analysis of neuroimaging datasets. Vol 2854 (2018).

88. J. M. Wardlaw, M. C. Valdés Hernández, S. Muñoz-Maniega, What are white matter hyperintensities made of? Relevance to vascular cognitive impairment. J. Am. Heart Assoc. 4, 001140 (2015).

89. F. J. Zhuang, Y. Chen, W. B. He, Z. Y. Cai, Prevalence of white matter hyperintensities increases with age. Neural Regen. Res. 13, 2141-2146 (2018).

90. D. M. Barch, et al., Function in the human connectome: Task-fMRI and individual differences in behavior. Neuroimage 80, 169-189 (2013).

91. S. Weintraub, et al., Cognition assessment using the NIH Toolbox. Neurology 80, S54-64 (2013).

92. D. S. Tulsky, et al., NIH Toolbox Cognition Battery (NIHTB-CB): The List Sorting Test to Measure Working Memory. J Int Neuropsychol Soc 20, 599-610 (2014).

93. J. P. Fortin, et al., Harmonization of cortical thickness measurements across scanners and sites. Neuroimage 167, 104-120 (2018).

94. R. Pomponio, et al., Harmonization of large MRI datasets for the analysis of brain imaging patterns throughout the lifespan. Neuroimage 208 (2020). 


\section{Figures and Tables}

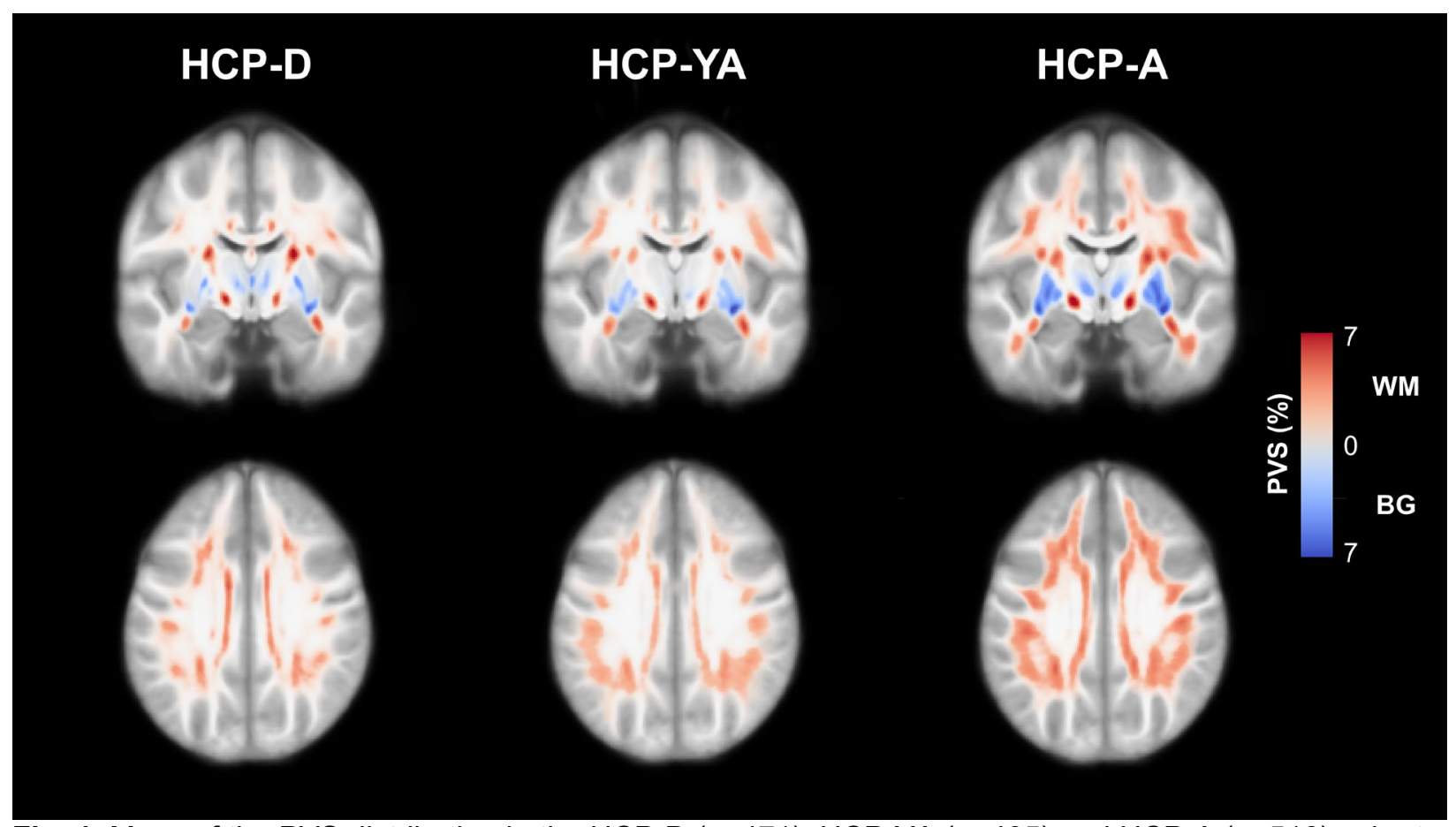

Fig. 1. Maps of the PVS distribution in the HCP-D ( $n=471)$, HCP-YA $(n=405)$ and HCP-A $(n=516)$ cohorts. Normative PVS maps were generated by registering each subject map to a population-averaged template and then averaging PVS segmentations across each group. The results are presented as the percentage of overlapping PVS across each group within the subcortical white matter (red) and basal ganglia (blue). PVS is prevalent in all HCP cohorts, including children and adolescents, and the apparent PVS burden increases with age in both the white matter and basal ganglia. 

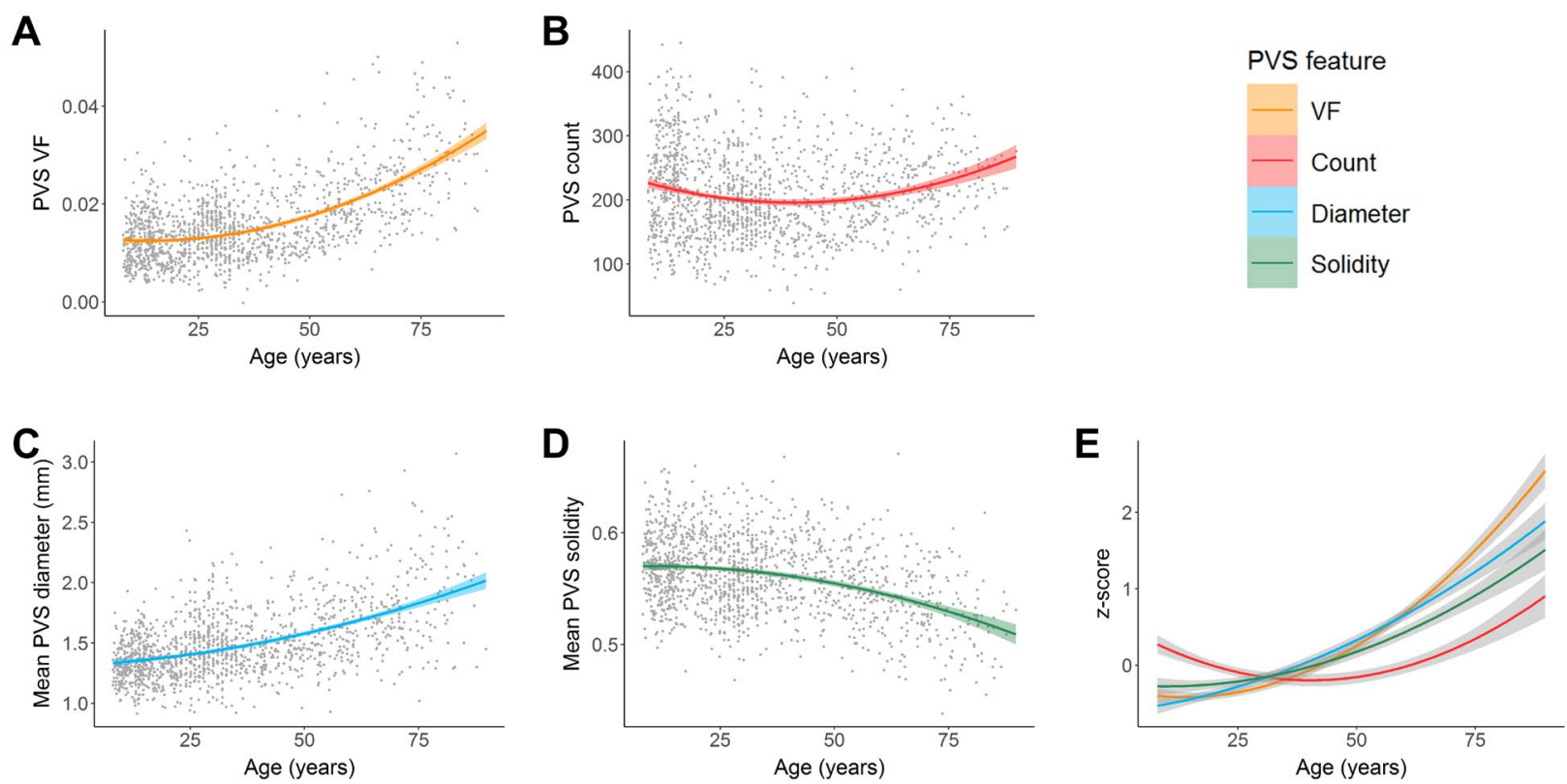

Fig. 2. Associations between PVS morphology and age in the basal ganglia. A quadratic curve provided the best fit model to describe the relationship between age and (A) PVS volume fraction, (B) count, (C) mean diameter and (D) mean solidity. (E) PVS features in the BG were standardized (z-scores) and plotted on the same normalized $y$-axis to enable comparison of feature differences with age over the lifespan ( $x$ axis). The mean PVS solidity curve was also inverted to show similarities and differences more easily. Within the BG, PVS volume fraction and mean PVS diameter undergo similar age-related trajectories, while mean PVS solidity and PVS count grow more slowly 

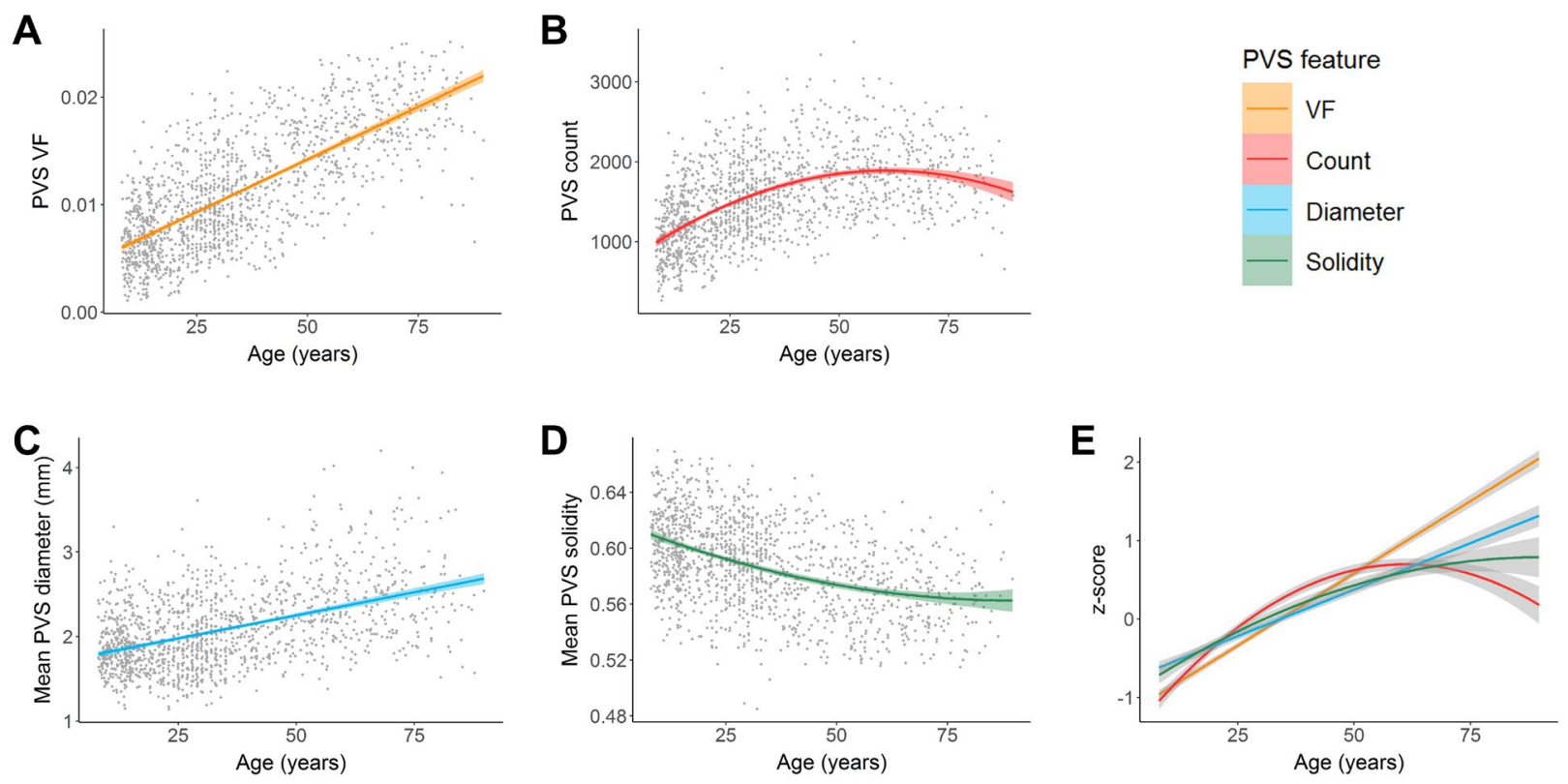

Fig. 3. Associations between PVS morphology and age in the subcortical white matter. Age was positively associated with (A) PVS volume fraction, (B) count and (C) mean diameter and negatively associated with (D) mean solidity. Age-related changes to the PVS volume fraction and mean diameter were best explained with a linear model, while age-related changes to the PVS count and mean solidity were best represented with a quadratic model. (E) PVS features in the subcortical white matter were standardized (z-scores) and plotted on the same normalized $y$-axis to enable comparison of feature differences with age over the lifespan (x-axis). The mean PVS solidity curve was also inverted to show similarities and differences more easily. Within the white matter, PVS count, mean PVS diameter and mean PVS solidity undergo slower age-related changes compared to PVS VF. PVS count, in particular, reaches peak counts at around 55 years of age. 
bioRxiv preprint doi: https://doi.org/10.1101/2022.01.25.475887; this version posted January 27, 2022. The copyright holder for this preprint (which was not certified by peer review) is the author/funder, who has granted bioRxiv a license to display the preprint in perpetuity. It is made available under aCC-BY-NC-ND 4.0 International license.
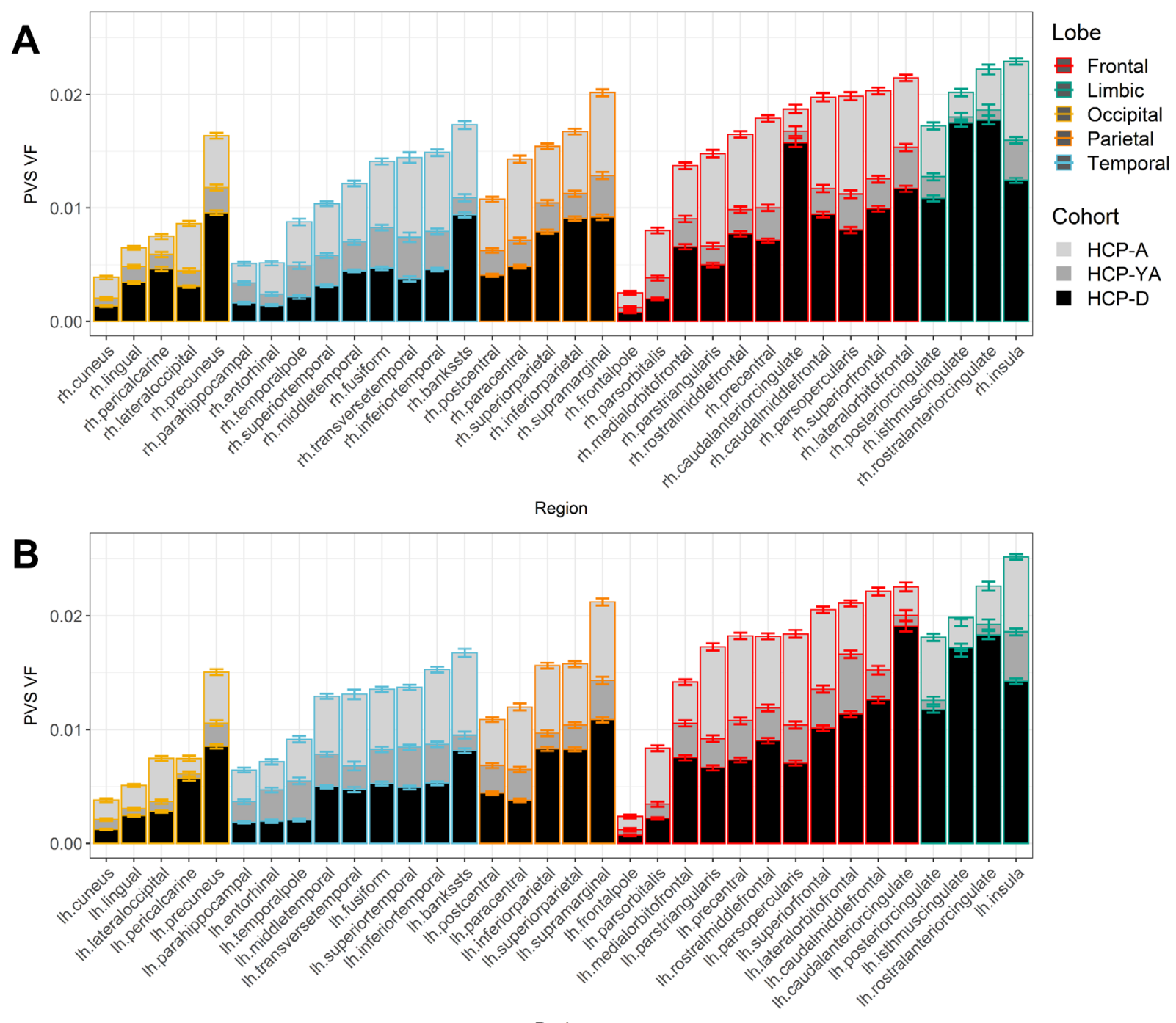

Region

Fig. 4. PVS VF varies across white matter regions. The mean PVS volume fraction across white matter regions in the $(A)$ left and $(B)$ right hemispheres are stratified by HCP cohort and ordered according to increasing PVS VF in the HCP-A cohort within major lobes. Occipital regions have the lowest PVS VF, while limbic and frontal regions have the highest. With some exceptions, regions with low PVS burden in aging subjects also tend to be low in young adults and developing subjects, while regions with high PVS burden in aging subjects tend to also be high in young adults and developing subjects. Bars represent standard error. 

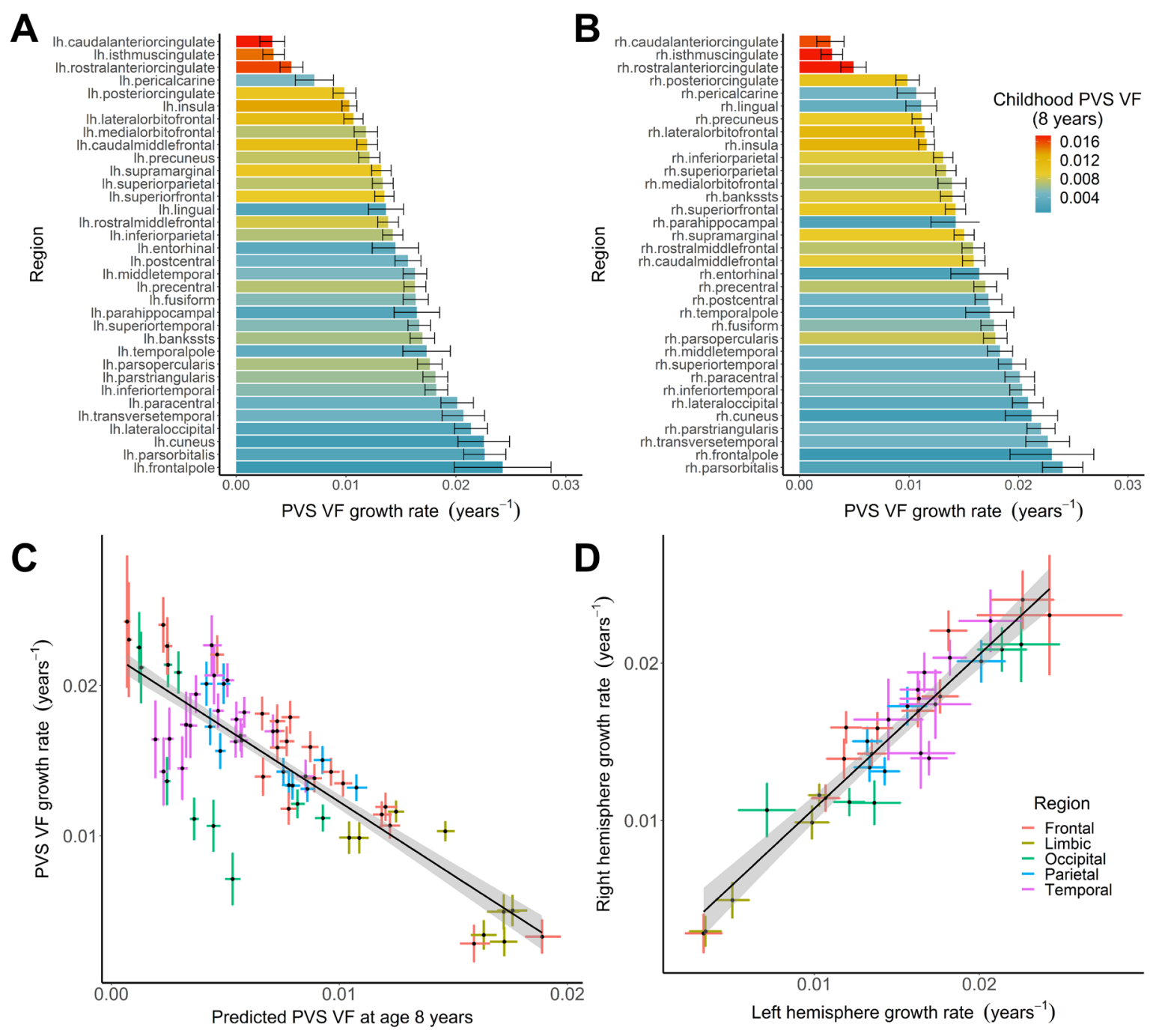

Fig. 5. The PVS VF growth rate across the lifespan is driven by PVS burden in childhood. Age-related changes to PVS volume fraction were fit with an exponential model to assess regional differences in PVS lifespan trajectories. Bars denoting the growth rate, $k$, for age-related changes to the PVS volume fraction are shown for the $(A)$ left and $(B)$ right white matter regions ordered from lowest to highest. The color of the bars indicate the estimated PVS VF at age 8 years. (C) The relationship between PVS VF predicted at 8 years of age (x-axis) and the PVS growth rate (y-axis) across the lifespan for bilateral white matter regions is shown. Each point reflects a single white matter region with the $95 \%$ confidence interval estimated with bootstrap resampling. PVS burden is inversely correlated with the PVS VF growth rate across the lifespan, with trends in major regional demarcations. Limbic regions with high PVS burden in childhood undergo minimal growth across the lifespan, while temporal regions have low PVS burden in childhood and undergo rapid growth across the lifespan. Occipital regions are an exception to this finding, which tend to have low PVS burden in childhood and undergo minimal growth across the lifespan. (D) The estimated growth rate for PVS VF in white matter regions are highly correlated between the left and right hemispheres. 


\section{A}
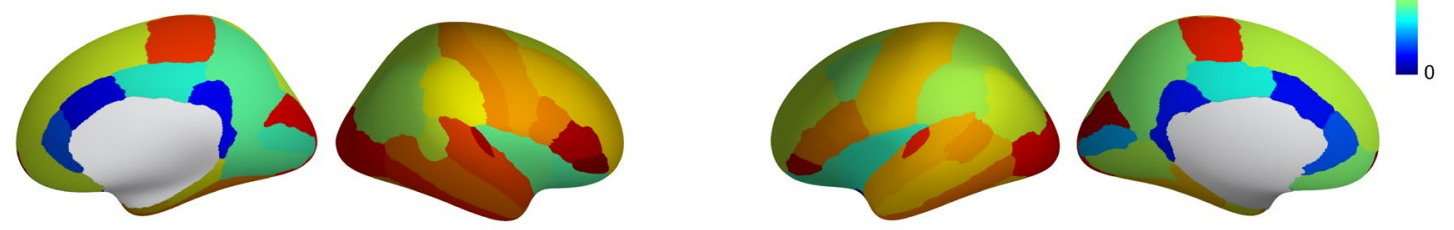

B
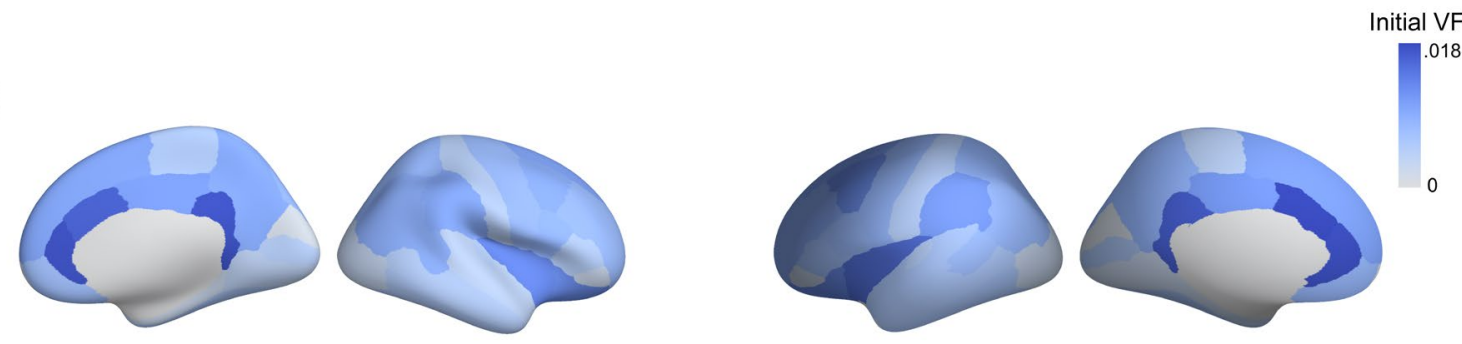

rowth rate, $k\left(\mathrm{~s}^{-1}\right)$
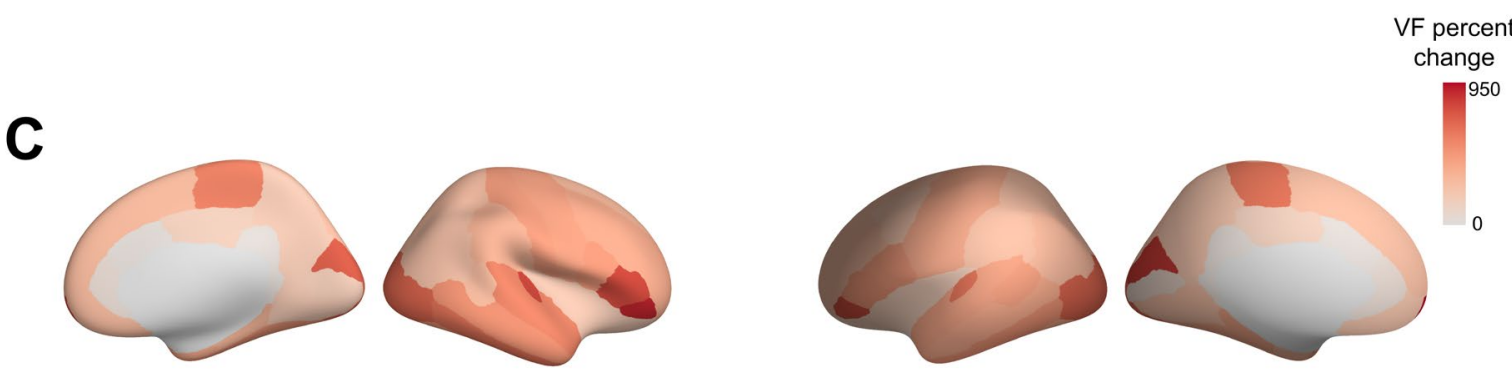

Fig. 6. Regional patterns of PVS VF trajectories across the lifespan. The (A) growth rate, (B) PVS VF at age 8 years and (C) percent change in PVS VF from age 8 to 89 years are shown for the 34 bilateral white matter regions. 
A

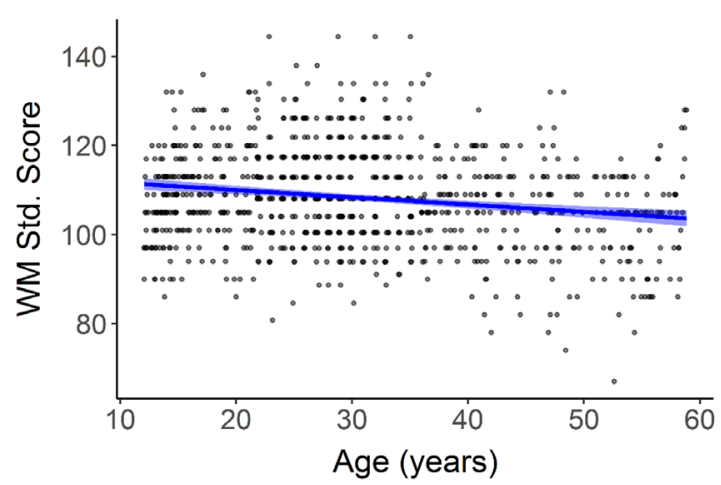

C

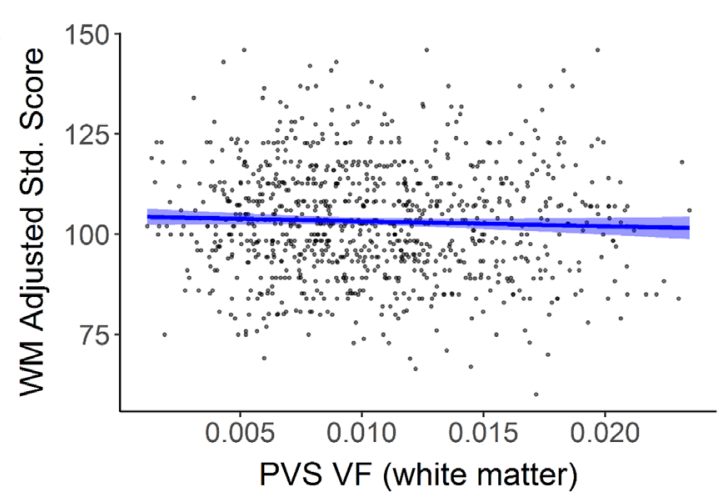

$\mathbf{E}$

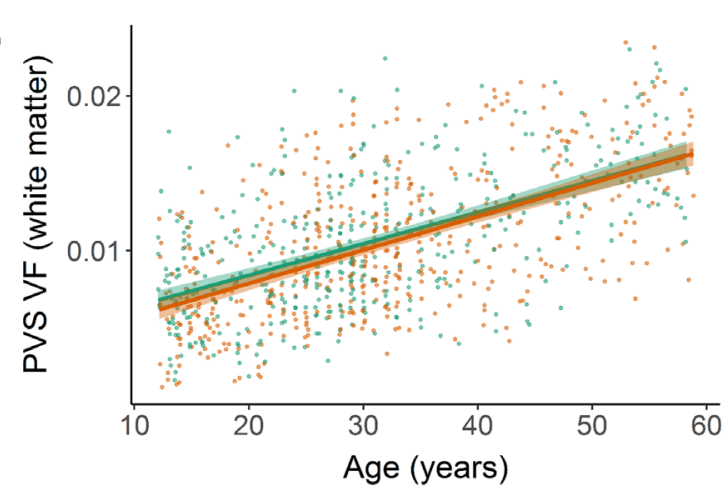

B

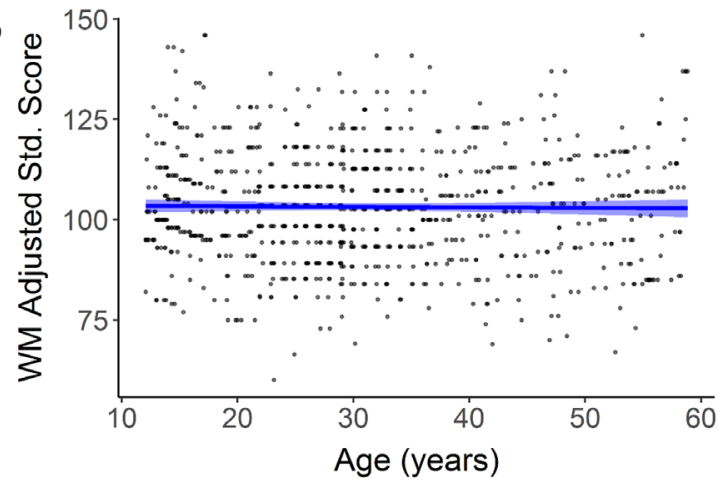

D

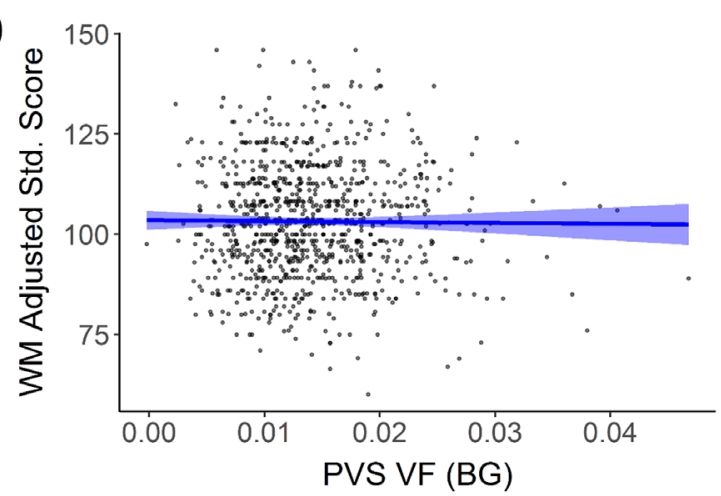

$\mathbf{F}$

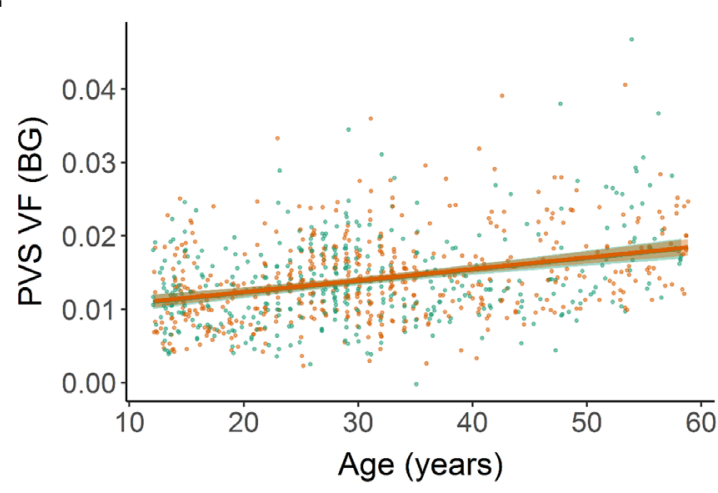

Working memory adjusted std. score: $\quad-<100 \quad->100$

Fig. 7. PVS burden does not influence working memory performance across the lifespan. (A) Age is associated with worse working memory performance assessed with the NIH Toolbox List Sorting Task. Utilizing (B) age-adjusted standardized scores, we sought to determine if PVS burden contributes to agerelated decline in working memory performance across the lifespan. PVS VF was not significantly associated with age-adjusted standardized scores in either the (C) white matter or (D) BG. Furthermore, when stratified by working memory performance (performance that is greater or lesser than the population mean), no significant differences in age-related PVS VF changes were observed between groups in either the $(E)$ white matter or $(F) B G$. 

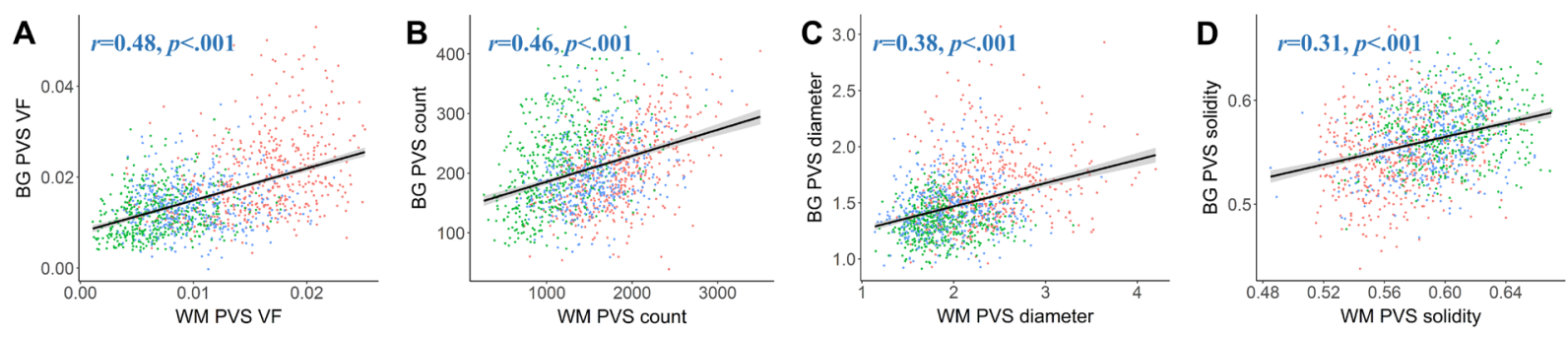

Fig. 8. Similar distributions of PVS morphological features are found in the BG and white matter. The relationship between PVS morphology between the white matter (x-axis) and BG (y-axis) are shown for (A) PVS VF, (B) PVS count, (C) mean PVS diameter and (D) mean PVS solidity are shown. A significant and positive relationship between PVS features in the white matter and basal ganglia were found for all PVS features, where subjects with low PVS burden in the white matter also tended to have low PVS burden in the $B G$, and vice versa. 
Table 1. Age distribution of HCP cohorts stratified by sex.

\begin{tabular}{lcccc} 
& & \multicolumn{4}{c}{ Age } \\
\cline { 3 - 5 } Dataset & $n$ & Mean (SD) & Range & $t$-statistic \\
\hline HCP-D & 471 & $14.36(3.77)$ & $8.08-21.92$ & -- \\
Males & 211 & $14.44(3.57)$ & $8.08-21.83$ & 0.43 \\
Females & 260 & $14.29(3.93)$ & $8.17-21.92$ & \\
\hline HCP-YA & 405 & $28.73(3.78)$ & $22-36$ & -- \\
Males & 179 & $27.89(3.64)$ & $22-36$ & $-4.07^{* * *}$ \\
Females & 226 & $29.40(3.78)$ & $22-36$ & -- \\
\hline HCP-A & 520 & $56.35(13.65)$ & $36-89.75$ & \multirow{2}{*}{0.47} \\
Males & 222 & $56.69(13.74)$ & $36.00-89.75$ & -- \\
Females & 298 & $56.11(13.59)$ & $36.17-87.75$ & \\
\hline Combined & 1396 & $34.17(20.07)$ & $8.08-89.75$ & 0.78 \\
$\quad$ Males & 612 & $33.70(20.16)$ & $8.08-89.75$ & \\
Females & 784 & $34.54(20.00)$ & $8.08-87.75$ & \\
\hline SD = standard deviation; ${ }^{* * *} \mathrm{p}<.001^{* *} \mathrm{p}<.01^{*} \mathrm{p}<.05$ &
\end{tabular}


Table 2. Association between PVS morphological features and age stratified by cohort

\begin{tabular}{|c|c|c|c|c|c|c|c|}
\hline \multirow[b]{2}{*}{ Variable } & \multirow[b]{2}{*}{ Cohort } & \multicolumn{3}{|c|}{ White matter } & \multicolumn{3}{|c|}{ Basal ganglia } \\
\hline & & $B$ & Std Error & $t$ & $B$ & Std Error & $t$ \\
\hline \multirow[t]{3}{*}{ VF } & HCP-D & $4.32 \times 10^{-4}$ & $3.65 \times 10^{-5}$ & $3.93^{* * *}$ & $2.11 \times 10^{-5}$ & $5.08 \times 10^{-5}$ & 0.41 \\
\hline & HCP-YA & $1.82 \times 10^{-4}$ & $4.85 \times 10^{-5}$ & $3.75^{\star *}$ & $4.45 \times 10^{-6}$ & $7.22 \times 10^{-5}$ & 0.06 \\
\hline & HCP-A & $1.78 \times 10^{-4}$ & $1.26 \times 10^{-5}$ & $14.07^{* * *}$ & $3.63 \times 10^{-4}$ & $1.99 \times 10^{-5}$ & $18.29^{* * *}$ \\
\hline \multirow[t]{3}{*}{ Count } & HCP-D & 32.58 & 4.59 & $7.09^{\star \star *}$ & -1.37 & 0.75 & -1.81 \\
\hline & HCP-YA & 15.19 & 5.34 & $2.85^{\star *}$ & 0.67 & 0.73 & 0.91 \\
\hline & HCP-A & 1.18 & 1.41 & 0.84 & 0.95 & 0.16 & $5.78^{\star \star *}$ \\
\hline \multirow[t]{3}{*}{ Diameter } & HCP-D & $6.69 \times 10^{-4}$ & $3.97 \times 10^{-3}$ & 0.17 & $3.36 \times 10^{-3}$ & $1.95 \times 10^{-3}$ & 1.72 \\
\hline & HCP-YA & $1.52 \times 10^{-2}$ & $5.41 \times 10^{-3}$ & $2.81^{*}$ & $6.18 \times 10^{-3}$ & $3.22 \times 10^{-3}$ & 1.92 \\
\hline & HCP-A & $1.18 \times 10^{-2}$ & $1.50 \times 10^{-3}$ & $7.88^{* * *}$ & $1.06 \times 10^{-2}$ & $8.81 \times 10^{-4}$ & $12.08^{* \star *}$ \\
\hline \multirow[t]{3}{*}{ Solidity } & HCP-D & $-3.76 \times 10^{-4}$ & $3.33 \times 10^{-4}$ & -1.13 & $1.42 \times 10^{-4}$ & $3.40 \times 10^{-4}$ & 0.42 \\
\hline & HCP-YA & $-1.18 \times 10^{-3}$ & $3.66 \times 10^{-4}$ & $-3.22^{* *}$ & $9.59 \times 10^{-5}$ & $4.15 \times 10^{-4}$ & 0.23 \\
\hline & HCP-A & $-3.68 \times 10^{-4}$ & $9.02 \times 10^{-5}$ & $-4.08^{* * *}$ & $-1.02 \times 10^{-3}$ & $9.55 \times 10^{-5}$ & $-10.70^{* * *}$ \\
\hline
\end{tabular}

The partial correlation coefficients for the influence of age on PVS morphological features after controlling for sex and scanner type for the white matter and BG stratified by cohort. Age-related changes to white matter PVS morphology were observed in all cohorts, while BG PVS morphological changes with age were only observed in the aging cohort. ${ }^{* * *} p<.001{ }^{* *} p<.01{ }^{*} p<.05$ 
Table 3. Quadratic model coefficients that relate PVS morphological features with age across the lifespan in white matter and basal ganglia

\begin{tabular}{|c|c|c|c|c|c|c|c|c|c|}
\hline & \multirow[b]{2}{*}{ Coefficient } & \multicolumn{4}{|c|}{ White matter } & \multicolumn{4}{|c|}{ Basal ganglia } \\
\hline & & $B$ & Std Error & $t$ & $R^{2}$ & $B$ & Std Error & $t$ & $R^{2}$ \\
\hline \multirow[t]{2}{*}{ VF } & Age & $1.96 \times 10^{-4}$ & $4.78 \times 10^{-6}$ & $40.93^{\star * *}$ & \multirow{2}{*}{0.55} & $-1.08 \times 10^{-4}$ & $3.40 \times 10^{-5}$ & $-3.18^{* *}$ & \multirow{2}{*}{0.37} \\
\hline & $\mathrm{Age}^{2}$ & -- & -- & -- & & $3.90 \times 10^{-6}$ & $3.97 \times 10^{-7}$ & $9.83^{* * *}$ & \\
\hline \multirow[t]{2}{*}{ Count } & Age & 39.26 & 2.45 & $16.03^{* * *}$ & \multirow{2}{*}{0.30} & -2.37 & 0.36 & $-6.55^{\star \star \star *}$ & \multirow{2}{*}{0.03} \\
\hline & $\mathrm{Age}^{2}$ & -0.32 & 0.03 & $-11.27^{* * *}$ & & $2.94 \times 10^{-2}$ & $4.23 \times 10^{-3}$ & $6.95^{\star \star *}$ & \\
\hline \multirow[t]{2}{*}{ Diameter } & Age & $1.09 \times 10^{-2}$ & $5.40 \times 10^{-4}$ & $20.18^{\star \star \star}$ & \multirow{2}{*}{0.23} & $2.04 \times 10^{-3}$ & $1.35 \times 10^{-3}$ & 1.51 & \multirow{2}{*}{0.29} \\
\hline & $\mathrm{Age}^{2}$ & -- & -- & -- & & $6.46 \times 10^{-5}$ & $1.58 \times 10^{-5}$ & $4.09^{* * *}$ & \\
\hline \multirow[t]{2}{*}{ Solidity } & Age & $-1.27 \times 10^{-3}$ & $1.59 \times 10^{-4}$ & $-7.96^{\star * *}$ & \multirow{2}{*}{0.20} & $1.87 \times 10^{-4}$ & $1.79 \times 10^{-4}$ & 1.05 & \multirow{2}{*}{0.14} \\
\hline & $\mathrm{Age}^{2}$ & $7.05 \times 10^{-6}$ & $1.86 \times 10^{-6}$ & $3.79^{* * *}$ & & $-9.56 \times 10^{-6}$ & $2.09 \times 10^{-6}$ & $-4.57^{* * *}$ & \\
\hline
\end{tabular}

Quadratic regression fits for the PVS morphological features in subcortical white matter and basal ganglia. The quadratic model did not provide the best fit for the white matter PVS VF and mean diameter and agerelated variance was better explained by a linear regression. ${ }^{* * *} p<.001{ }^{* *} p<.01{ }^{*} p<.05$ 
bioRxiv preprint doi: https://doi.org/10.1101/2022.01.25.475887; this version posted January 27, 2022. The copyright holder for this preprint (which was not certified by peer review) is the author/funder, who has granted bioRxiv a license to display the preprint in perpetuity. It is made available under aCC-BY-NC-ND 4.0 International license.

Table 4. Sex differences in PVS morphological features stratified by HCP cohort

\begin{tabular}{|c|c|c|c|c|c|c|c|}
\hline \multirow[b]{3}{*}{ Feature } & \multirow[b]{3}{*}{ Cohort } & \multicolumn{3}{|c|}{ White matter } & \multicolumn{3}{|c|}{ BG } \\
\hline & & & Females & Males & & Females & Males \\
\hline & & $t$ & Mean (SD) & Mean (SD) & $t$ & Mean (SD) & Mean (SD) \\
\hline \multirow[t]{3}{*}{ VF } & HCPD & $6.67^{* * *}$ & $6.25 \times 10-3\left(2.87 \times 10^{-3}\right)$ & $8.16 \times 10-3\left(3.29 \times 10^{-3}\right)$ & $6.33^{* * *}$ & $1.05 \times 10-2\left(4.89 \times 10^{-3}\right)$ & $1.34 \times 10-2\left(5.14 \times 10^{-3}\right)$ \\
\hline & HCPYA & $2.98^{\star *}$ & $9.68 \times 10-3\left(3.51 \times 10^{-3}\right)$ & $1.05 \times 10-2\left(3.87 \times 10^{-3}\right)$ & -1.08 & $1.57 \times 10-2\left(4.56 \times 10^{-3}\right)$ & $1.51 \times 10-2\left(5.43 \times 10^{-3}\right)$ \\
\hline & $\mathrm{HCPA}$ & 0.08 & $1.55 \times 10-2\left(4.66 \times 10^{-3}\right)$ & $1.57 \times 10-2\left(4.42 \times 10^{-3}\right)$ & $7.08^{* * *}$ & $1.74 \times 10-2\left(8.03 \times 10^{-3}\right)$ & $2.24 \times 10-2\left(9.47 \times 10^{-3}\right)$ \\
\hline \multirow[t]{3}{*}{ Count } & HCPD & $2.06^{*}$ & $1101(404)$ & $1374(441)$ & $4.16^{\star \star \star}$ & $181(69)$ & $245(68)$ \\
\hline & HCPYA & -1.37 & 1489 (431) & $1411(382)$ & -1.06 & $209(43)$ & $227(64)$ \\
\hline & $\mathrm{HCPA}$ & 1.67 & $1713(396)$ & $2045(480)$ & $5.51^{* \star *}$ & $177(50)$ & $235(62)$ \\
\hline \multirow{3}{*}{$\begin{array}{c}\text { Diameter } \\
(\mathrm{mm})\end{array}$} & HCPD & $3.68^{* * *}$ & $1.79(0.39)$ & $1.93(0.42)$ & 0.69 & $1.31(0.23)$ & $1.33(0.23)$ \\
\hline & HCPYA & $3.31^{* *}$ & $1.92(0.38)$ & $2.04(0.43)$ & -0.63 & $1.45(0.24)$ & $1.43(0.22)$ \\
\hline & HCPA & -1.96 & $2.39(0.45)$ & $2.33(0.41)$ & $3.20^{\star \star}$ & $1.64(0.28)$ & $1.71(0.29)$ \\
\hline \multirow[t]{3}{*}{ Solidity } & HCPD & $-4.43^{\star \star \star}$ & $0.61(0.03)$ & $0.60(0.03)$ & $-4.54^{\star * *}$ & $0.58(0.03)$ & $0.57(0.03)$ \\
\hline & HCPYA & 1.23 & $0.59(0.03)$ & $0.59(0.03)$ & $-2.59^{* *}$ & $0.56(0.03)$ & $0.56(0.03)$ \\
\hline & HCPA & $-3.17^{* *}$ & $0.57(0.03)$ & $0.57(0.03)$ & $-6.32^{\star * *}$ & $0.56(0.03)$ & $0.54(0.04)$ \\
\hline
\end{tabular}

Main effect of sex on PVS morphological features in subcortical white matter and BG per HCP cohort after controlling for the effects of sex. PVS count is additionally normalized by intra-cranial volume. SD = standard deviation; ${ }^{* * *} p<.001^{* *} p<.01{ }^{*} p<.05$ 